ARTICLE

\title{
An inducible circular RNA circKcnt2 inhibits ILC3 activation to facilitate colitis resolution
}

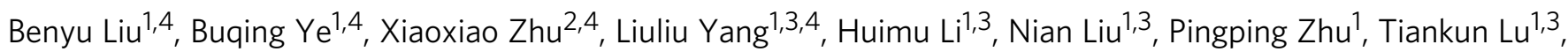
Luyun $\mathrm{He}^{1,3}$, Yong Tian (1) ${ }^{2,3 凶} \&$ Zusen Fan (1) ${ }^{1,3 凶}$

Group 3 innate lymphoid cells (ILC3) are an important regulator for immunity, inflammation and tissue homeostasis in the intestine, but how ILC3 activation is regulated remains elusive. Here we identify a new circular RNA (circRNA) circKcnt2 that is induced in ILC3s during intestinal inflammation. Deletion of circKcnt2 causes gut ILC3 activation and severe colitis in mice. Mechanistically, circKcnt2, as a nuclear circRNA, recruits the nucleosome remodeling deacetylase (NuRD) complex onto Batf promoter to inhibit Batf expression; this in turn suppresses II17 expression and thereby ILC3 inactivation to promote innate colitis resolution. Furthermore, $\mathrm{Mbd3}^{-/-}$Rag1 $^{-/-}$and circKcnt2 ${ }^{-/-}$Rag $1^{-/-}$mice develop severe innate colitis following dextran sodium sulfate (DSS) treatments, while simultaneous deletion of Batf promotes colitis resolution. In summary, our data support a function of the circRNA circKcnt2 in regulating ILC3 inactivation and resolution of innate colitis.

\footnotetext{
${ }^{1}$ Key Laboratory of Infection and Immunity of CAS, CAS Center for Excellence in Biomacromolecules, Institute of Biophysics, Chinese Academy of Sciences, 100101 Beijing, China. ${ }^{2}$ Key Laboratory of RNA Biology, Institute of Biophysics, Chinese Academy of Sciences, 100101 Beijing, China. ${ }^{3}$ University of Chinese Academy of Sciences, 100049 Beijing, China. ${ }^{4}$ These authors contributed equally: Benyu Liu, Buqing Ye, Xiaoxiao Zhu, Liuliu Yang. ${ }^{\circledR e m a i l: ~ y t i a n @ i b p . a c . c n ; ~}$ fanz@moon.ibp.ac.cn
} 
nnate lymphoid cells (ILCs) mainly reside in mucosal surfaces and play a critical role in sustaining mucosal integrity and promoting lymphoid organogenesis ${ }^{1,2}$. According to their specific fate-decision transcription factors (TFs) and secreted cytokines, ILCs can be classified into three subgroups, including group 1 ILC (ILC1s), group 2 ILC (ILC2s), and group 3 ILC (ILC3s) $)^{1,3}$. Among them, ILC3s producing interleukin-22 (IL-22), IL-17, and interferon- $\gamma$ (IFN- $\gamma)$ are essential for maintenance of intestinal homeostasis ${ }^{4,5}$. Accumulating evidence indicates ILC3s are implicated in intestinal diseases, such as inflammatory bowel disease (IBD) ${ }^{6}$. Increased secretion of IL- 22 by ILC3s facilitates resolution of microbial infection and tissue injury in the gut ${ }^{5,7}$. Nevertheless, excessive activation of ILC3s has been reported to induce colitis and cancer ${ }^{6,8}$. ILC3s in Rag (VDJ recombination activation gene)-deficient mice can cause innate colitis after antiCD40 or dextran sodium sulfate (DSS) treatment ${ }^{9,10}$. In fact, ILC3 activation and inactivation in vivo are finely regulated to maintain gut homeostasis. For example, IL-23-induced STAT3 phosphorylation is a major pathway for ILC3 activation ${ }^{11}$. Treg cells can suppress ILC3-mediated colitis in an IL-10-independent fashion $^{12}$. We previously showed that ILCregs inhibit ILC3 activation via secretion of IL-10 (ref. ${ }^{13}$ ). However, whether and how noncoding RNA modulates ILC3 activity are unclear.

Circular RNAs (circRNAs), a class of noncoding RNAs, are characterized by a covalent bond linking the $3^{\prime}$ and $5^{\prime}$ ends formed by back-splicing ${ }^{14}$. CircRNAs were previously considered as byproducts of RNA splicing ${ }^{15}$. CircRNAs are generated by exons, introns, or exon-introns ${ }^{16}$. It has been reported that circRNAs extensively exist in various tissues and their expression profiles exhibit tissue and cell-type-specific patterns ${ }^{17,18}$. Moreover many circRNAs are highly conserved across species ${ }^{19}$. CircRNAs play critical roles in a variety of biological processes. For instance, ciRS-7 and Sry serve as miRNA sponges to exert their functions in brain ${ }^{20}$. Some exon-intronic circRNAs are located in the nucleus that are involved in the regulation of gene transcription by interacting with RNA polymerase II (ref. ${ }^{21}$ ). CircRNAs are also implicated in the modulation of tumorigenesis ${ }^{22}$. We previously showed that a circRNA cia-cGAS associates with DNA sensor cGAS in the nuclei of hematopoietic stem cells (HSC) to block its synthase activity, maintaining HSC hemeostasis ${ }^{18}$. We recently demonstrated that circPan3 can promote the self-renewal of intestinal stem cells through IL-13 produced by niche ILC2s ${ }^{18,23}$.

Basic leucine zipper transcription factor ATF-like (Batf) belongs to the activator protein 1 (AP-1) family of $\mathrm{TFs}^{24}$. Accumulating evidence shows that Batf modulates adaptive immunity as a critical regulator. Batf participates in the regulation of differentiation of Th17, Th9, and follicular helper T (Tfh) cells ${ }^{25}$. Batf also modulates the class-switch recombination of $\mathrm{B}$ cells ${ }^{26}$. In addition, Batf deficiency reduces production of IFN- $\gamma$ by $\mathrm{CD}^{+} \mathrm{T}$ cells ${ }^{27}$. Batf upregulation can suppress the effector function of exhausted CD8 ${ }^{+}$ $\mathrm{T}$ cells ${ }^{28}$. Moreover, Batf is also involved in the regulation of regulatory $\mathrm{T}\left(\mathrm{T}_{\mathrm{reg}}\right)$ cell development and maintanance ${ }^{29,30}$. However, the role of Batf in ILCs remains elusive.

Here we show that the circRNA circKcnt2 (originating from Knct2 gene transcript; gene symbol, mmu_circRNA_012594 or mmu_circ_0000084 in circBase) is highly induced in activated ILC3s during intestinal innate inflammation. CircKcnt2 recruits the NuRD complex onto Batf promoter to suppress its expression, which inhibits ILC3 activation to promote innate colitis resolution. These results discover a previously unidentified contribution of circRNA to the regulation of colitis.

\section{Results}

CircKcnt2 is highly induced in ILC3s during inflammation. To explore how circRNAs regulate ILC3 function in innate colitis, we isolated ILC3s from DSS-treated and control Rag1-/- mice, followed by circRNA microarray analysis. With DSS treatment, many differentially expressed circRNAs appeared in Rag1-IILC3s (Fig. 1a). We then chose top ten most upregulated circRNAs for validation. Their identities as circRNAs were further verified by PCR (Supplementary Fig. 1a) and RNase R digestion (Supplementary Fig. 1b). These ten circRNAs were really highly expressed in ILC3s isolated from DSS-treated Rag $1^{-1}$ mice through quantitative reverse transcriptase PCR (qRT-PCR) analysis (Fig. 1b). To further determine the functions of these ten circRNAs in ILC3s, we isolated ILC3s from DSS-treated Rag1-1mice and silenced them by shRNAs targeting head-to-tail regions, and then transplanted these cells into $\operatorname{Ragl}^{-1}-I l 2 \mathrm{rg}^{-1-}$ mice, followed by DSS treatment (Fig. 1c). Silencing efficiencies of these ten circRNAs with shRNAs were validated by qRT-PCR (Supplementary Fig. 1c). However, circRNA depletion did not affect the expression levels of their cognate linear transcripts (Supplementary Fig. 1d). Of these ten circRNAs, circKcnt2 depletion caused more aggravated colon injury, lymphocyte infiltration (Fig. 1d), and more severe innate colitis (Fig. 1e). CircPou2f1 or circCcdc141 knockdown caused slight intestinal inflammation (Fig. 1d), but they did not cause statistical significant innate colitis compared to shCtrl treated mice (Fig. 1e). In addition, circAkap6 or circMyh10 depletion caused slight innate colitis (Fig. 1e). Here we focused on the role of circKcnt2 in the regulation of innate colitis.

CircKcnt2 is formed by back-splicing of Kcnt2 transcripts from exon 4 to exon 8 . We confirmed the back-splicing site through sequencing entire circKcnt2 transcript (Supplementary Fig. 1e). Of note, circKcnt 2 was highly and specifically expressed in intestinal ILC3s through northern blot and qRT-PCR analyses (Fig. 1f, g). In addition, circKcnt2 expression was gradually elevated in ILC3s during DSS treatment, reaching a peak level on day 6 (Fig. 1h). CircKcnt2 was mainly distributed in the nuclei of ILC3s isolated from DSS-treated Rag1-1- mice (Fig. 1i, Supplementary Fig. 1f). Finally, circKcnt 2 was highly conserved across species (Supplementary Fig. 1g). The back-splicing site of human circKCNT2 was verified by DNA sequencing (Supplementary Fig. 1h). Collectively, circKcnt2 is highly induced in ILC3s during innate colitis.

CircKcnt2 knockout induces ILC3 activation and colitis. To

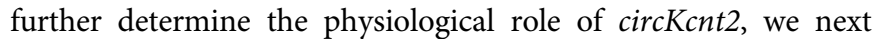
sought to generate circKcnt2-deficient mice. The complementary elements flanking circRNA sequences were essential for their formation ${ }^{16}$. We then screened out the complementary sequences in the introns flanking circKcnt 2 and analyzed their conservation across species (Supplementary Fig. 2a-c). Through minigene assay, we verified that the complementary sequences against circKcnt2 were required for its formation (Supplementary Fig. 2d). We next generated circKcnt2 knockout (circKcnt2-l-) mice through deleting the complementary intron sequences of genome with CRISPR/CAS9 technology (Supplementary Fig. 2e). CircKcnt2 was successfully deleted in circKcnt $2^{-1-}$ mice, while its maternal gene Kcnt2 mRNA was not affected (Supplementary Fig. 2f-h). Furthermore, Kcnt2 expression was similar in circKcnt $2^{-1-}$ mice versus WT mice (Supplementary Fig. 2h, i).

We firstly tested the effect of circKcnt2 on ILC development. We found that circKcnt2-I- mice exhibited comparable numbers of ILC progenitors, including CLPs, CHILPs, and ILCPs in bone marrow (BM) to circKcnt $2^{+/+}$littermate WT mice (Supplementary Fig. 3a-c). Moreover, the numbers of NKs, ILC1s, ILC2s, and ILC3s were also not affected by circKcnt2 deletion (Supplementary Fig. 3d-h). We thus wanted to determine whether circKcnt2 deletion affected ILC3 activation during colitis induction. 
a

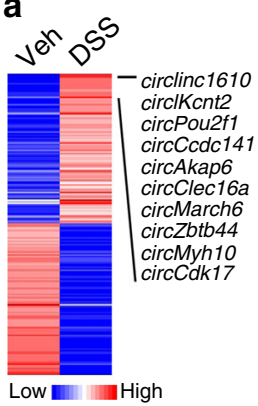

b

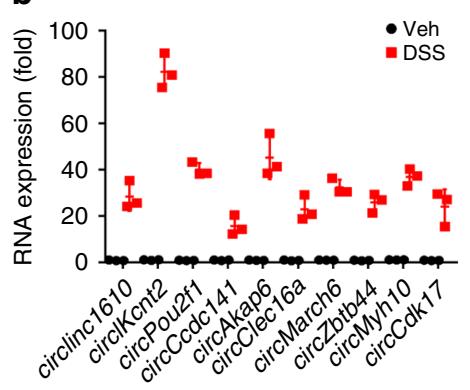

C

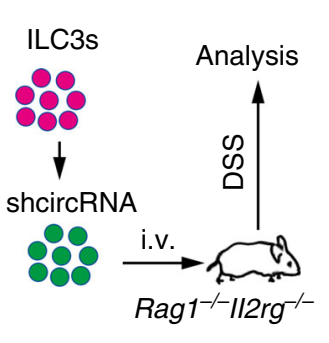

e

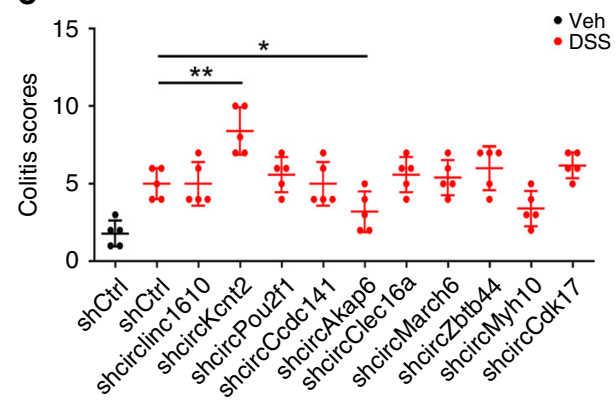

d

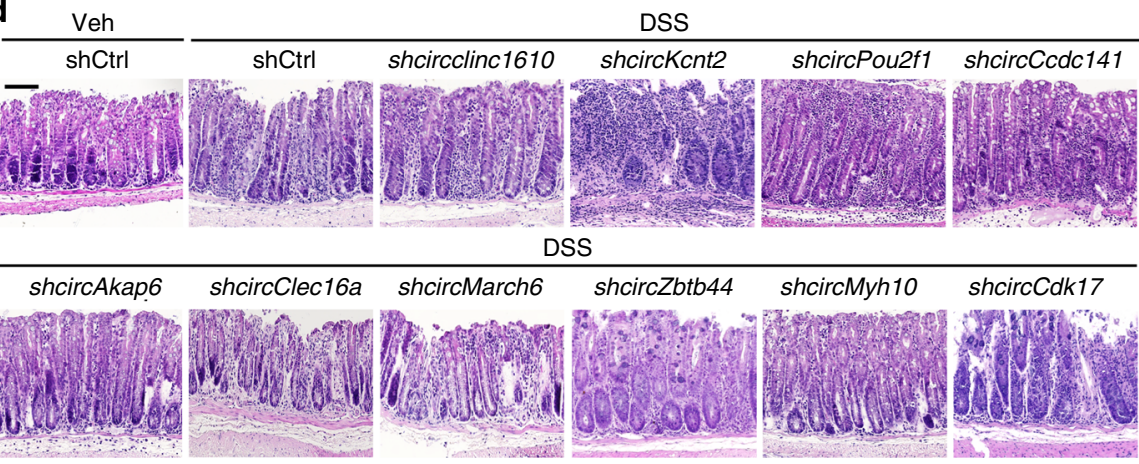

g

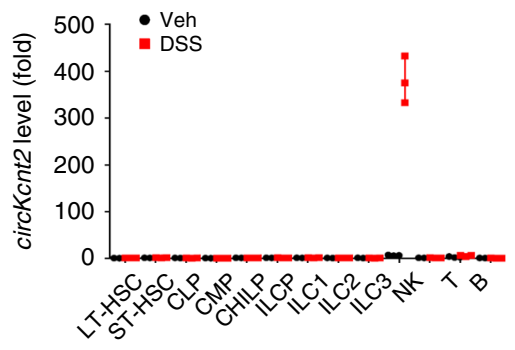

f

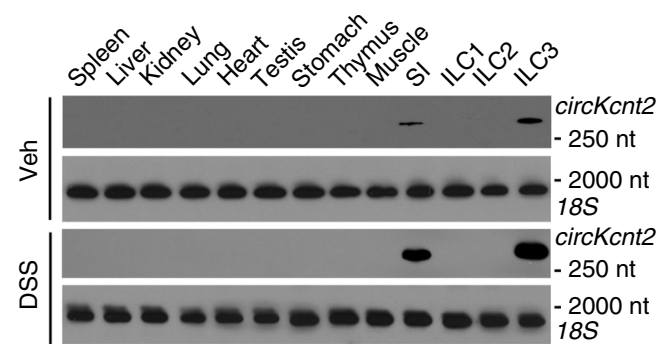

h

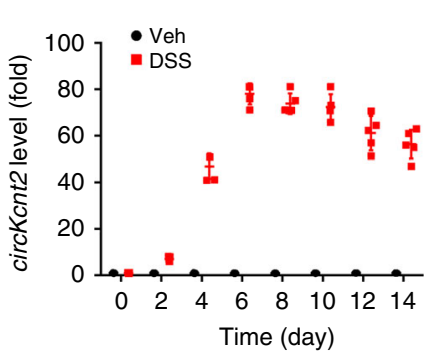

i

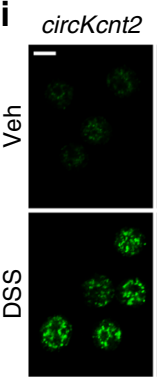

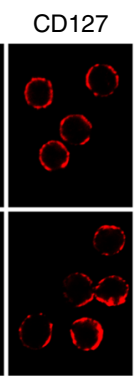

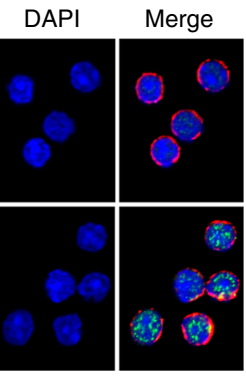

Fig. 1 CircKcnt2 is highly induced in ILC3s during intestinal inflammation. a Heatmap of differentially expressed circRNAs (100 most upregulated and 100 most downregulated) in small intestinal ILC3s (Lin-CD45low CD90high) from Rag1-/- mice treated with or without DSS. ILC3s in intestines were isolated by FACS, followed by circRNA microarray analysis. Veh vehicle. b qRT-PCR analysis for top ten upregulated circRNAs in small intestinal ILC3s from DSStreated Rag1 ${ }^{-1}$ - mice. $n=3$ independent samples. c ILC3s isolated from DSS-treated Rag1-/- mice were infected with lentiviruses carrying shRNAs

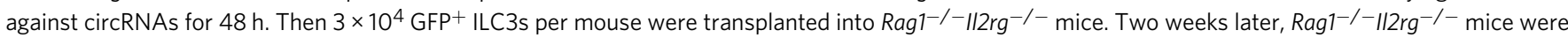
treated with DSS, followed by analysis. $\mathbf{d}$ H\&E staining of colons from transplanted Rag ${ }^{-1} / 1 / 2 \mathrm{rg}^{-/-}$mice as treated in c. Scale bar, $100 \mu \mathrm{m}$. e Colitis scores of indicated mice after DSS treatment as in $\mathbf{c}\left({ }^{\star} P=0.0399 ;{ }^{\star \star} P=0.0031\right) . n=5$ for each group. $\mathbf{f}$ Northern blotting analysis for circKcnt2 expression in different tissues from Rag1 ${ }^{-1-}$ mice treated with or without DSS. $\mathbf{g}$ qRT-PCR analysis for circKcnt2 expression in indicated hematopoietic cells isolated from WT mice treated with or without DSS. $n=3$ independent samples. h CircKcnt2 expression in ILC3s from Rag $1^{-1-}$ mice treated with $3 \%$ DSS was analyzed at different time points. $n=5$ independent samples. i ILC3s were isolated from Rag1-/- mice treated with $3 \%$ DSS and then circKcnt2 expression was detected by fluorescence in situ hybridization (FISH). Biotin-labeled head-to-tail RNA probes were in green. Nuclei were counterstained with DAPI. Scale bar, $5 \mu \mathrm{m} .{ }^{\star} P<0.05$ and ${ }^{\star \star \star} P<0.001$. Data were analyzed by an unpaired Student's $t$-test and shown as means \pm SD. Data are representative of at least three independent experiments. Source data are provided as a Source Data file.

We then generated circKcnt2-I- $\mathrm{Rag} 1^{-1-}$ mice through crossing circKcnt2 ${ }^{-1-}$ mice with $R a g 1^{+/-}$mice, followed by DSS treatment. We found that ILC3s of circKcnt2 $2^{-1-R a g 1^{-1-}}$ mice expressed much more amount of Il17a after DSS treatment compared to circKcnt2 ${ }^{+/+}$Rag1 $^{-1-}$ mice (Fig. 2a). Furthermore, IL-17 ${ }^{+}$ILC3 numbers in DSS-treated circKcnt2 ${ }^{-1-}$ Rag1 ${ }^{-1-}$ mice were much greater than those of circKcnt2 $2^{+/+}$Rag $^{-1-}$ mice (Fig. 2b). Of note, IL-17 ${ }^{+}$ILC3s of circKcnt2 $2^{-1}$ Rag1 $1^{-1}$ mice did not undergo cell death (Supplementary Fig. 3i). In addition, MFI of $\mathrm{IL}-17^{+}$ILC3s from DSS-treated circKcnt2 ${ }^{-1-}$ Rag1 $1^{-1}$ mice was much higher than that of $\operatorname{circKcnt2^{+/}}$ Rag1 $^{-/-}$mice (Fig. 2c), suggesting that circKcnt2 $2^{-1-} \mathrm{Rag1}^{-1-}$ ILC3s endowed IL-17-producing potential. In fact, circKcnt2 ${ }^{-I-} \mathrm{Rag1}^{-1-}$ ILC3s produced more substantial amounts of IL-17 than $c$ ircKcnt $2^{+/+}$Rag1 $^{-/-}$ILC3s (Fig. 2d). We next overexpressed circKcnt2 in circKcnt2-l- ILC3s for rescue assay. As expected, circKcnt2 was really restored in circKcnt2-1ILC3s (Supplementary Fig. 3j). Restoration of circKcnt2 in circKcnt $2^{-/-} \mathrm{Rag1}^{-/-}$ILC3s could inhibit IL-17 generation (Supplementary Fig. 3k). These data indicate that circKcnt2 suppresses IL-17 production in ILC3s.

It has been reported that IL-17 produced by ILC3s is an important regulator for innate colitis ${ }^{8,13}$. We next sought to explore whether circKcnt2 participated in innate inflammation via modulating ILC3 activation. We noticed that $\operatorname{circKcnt2^{-I}} \mathrm{Rag1}^{-1-}$ mice lost more body weight than $\operatorname{circKcnt} 2^{+/+} \mathrm{Rag1}^{-/-}$mice after DSS treatment (Fig. 2e). And circKcnt $2^{-I-} \mathrm{Rag1}^{-I-}$ mice displayed 
a

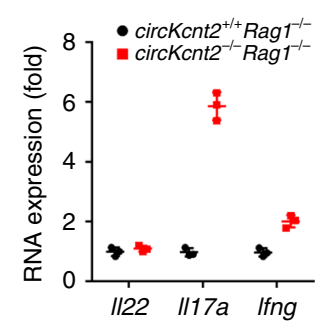

b

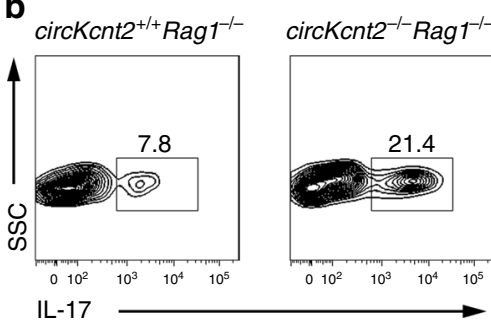

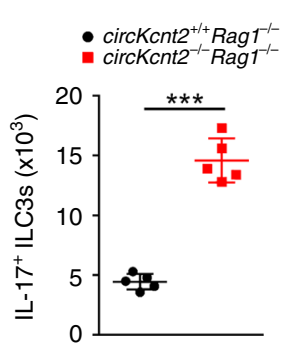
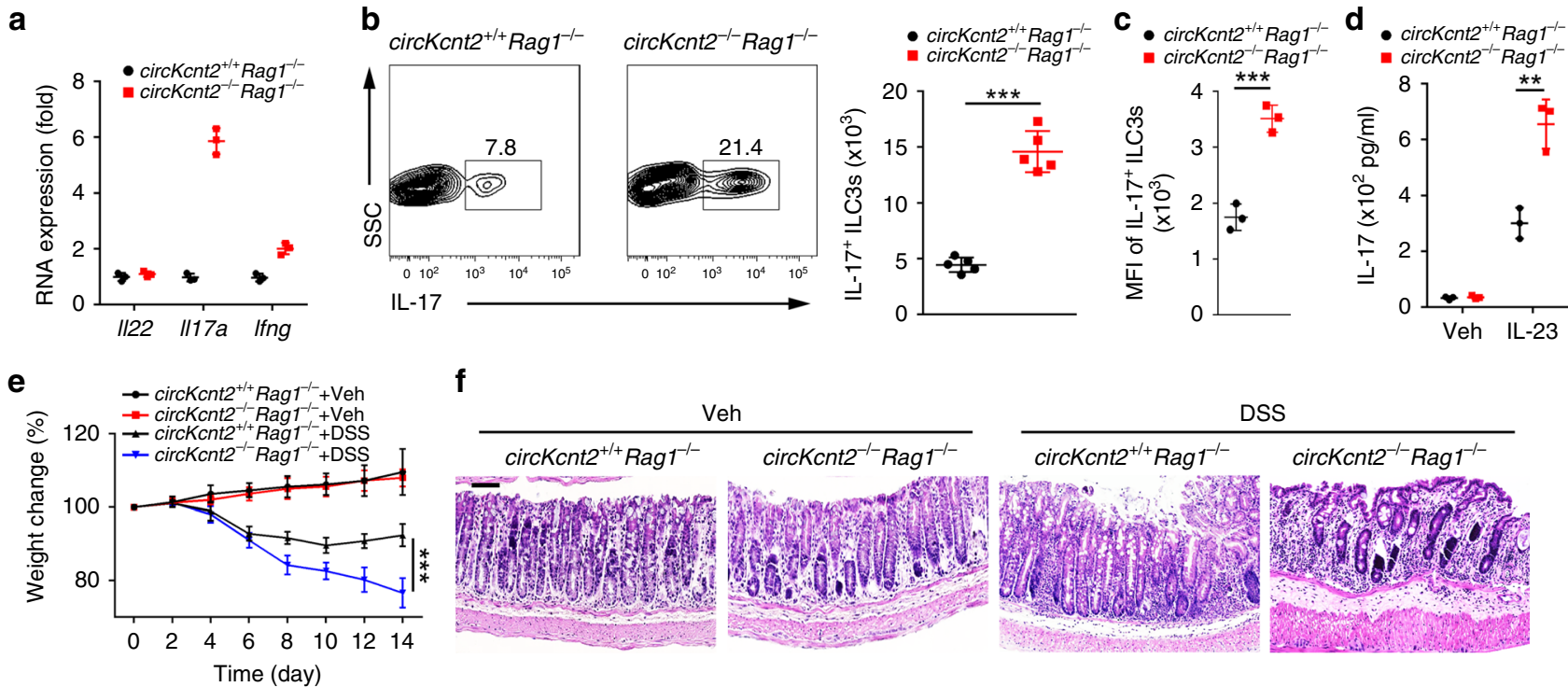

f
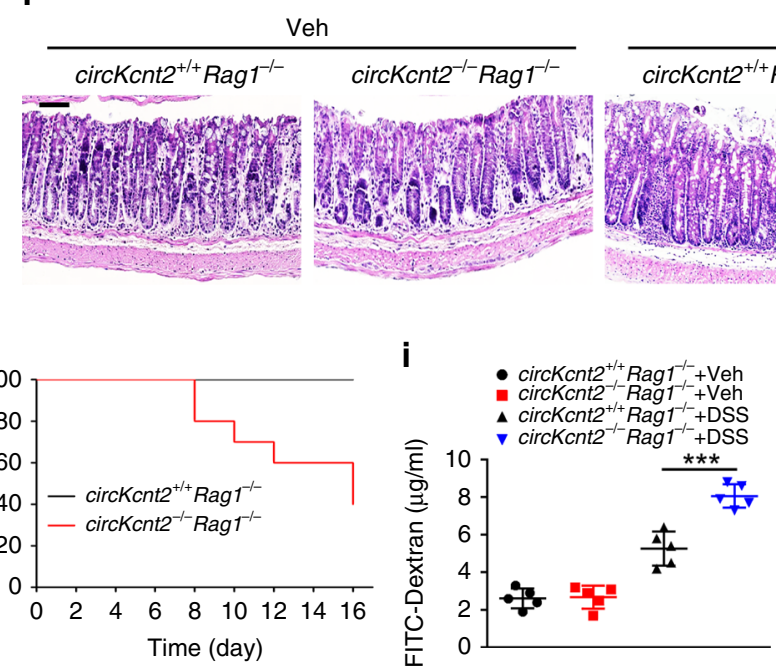

h

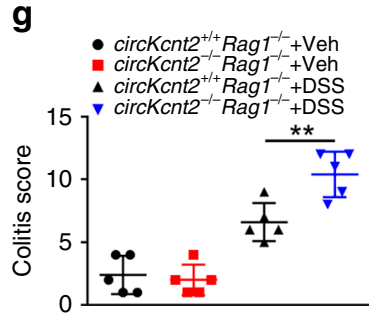

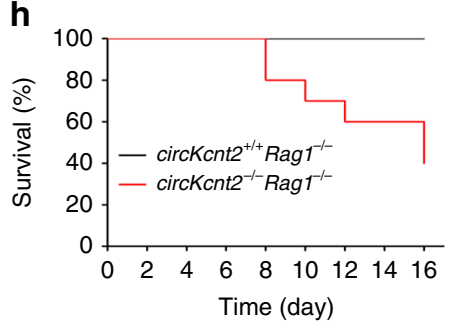

|

$\mathbf{k}$
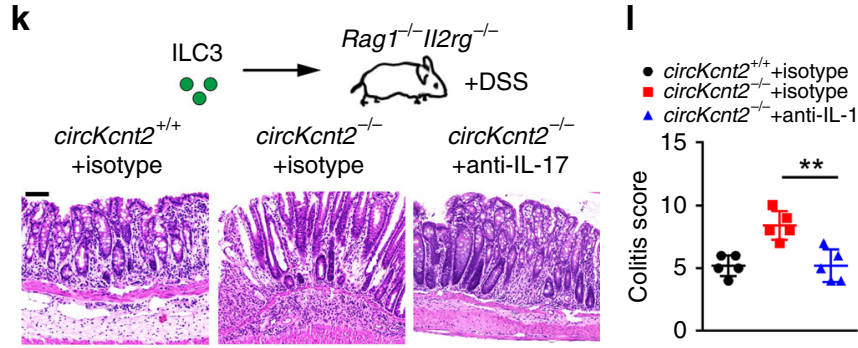

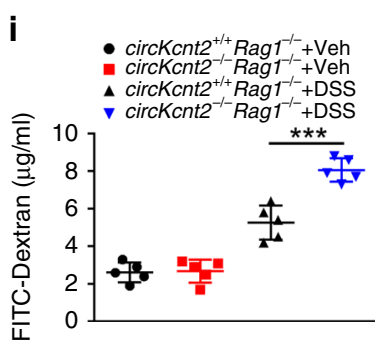

\section{m}
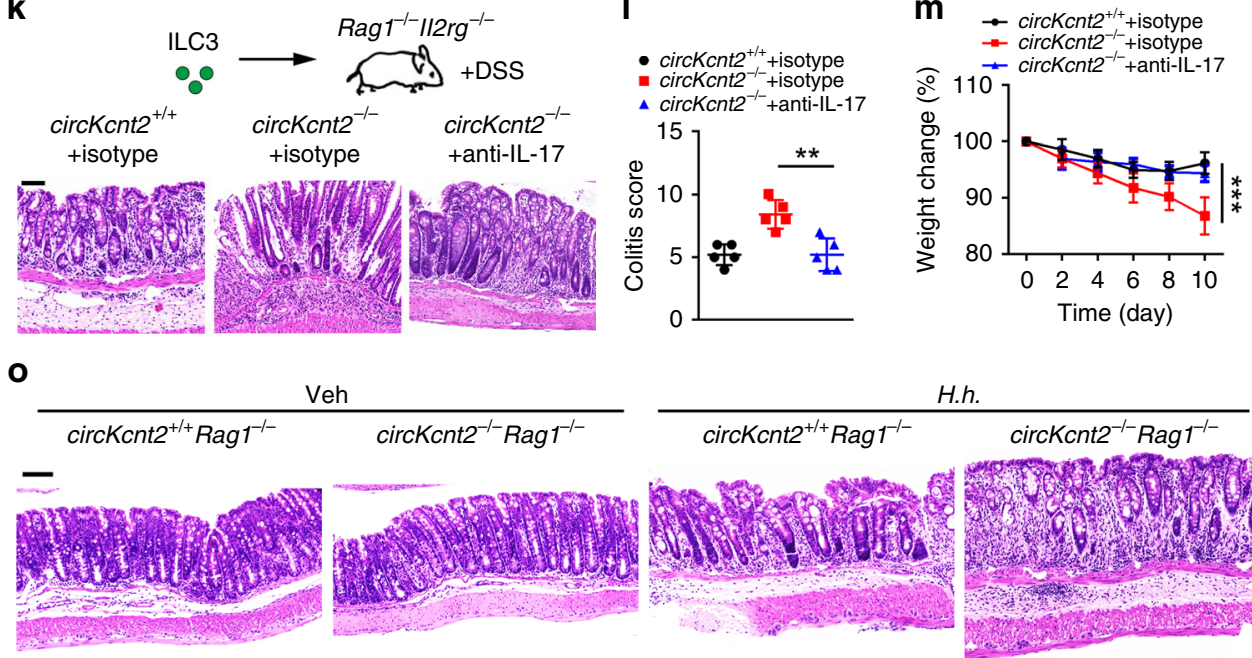

eh circKcnt2 ${ }^{-/-}$Rag1 $^{-/-}$

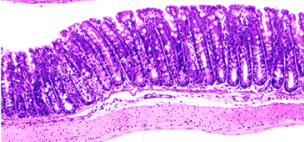

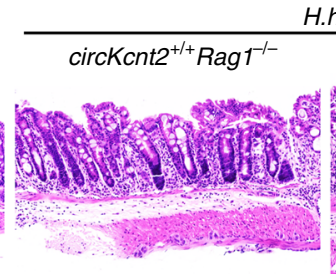

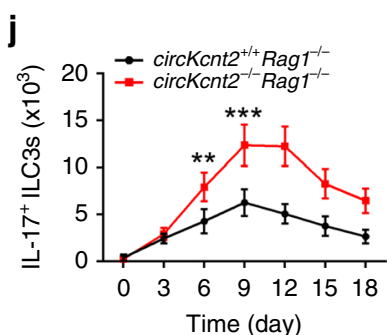

n

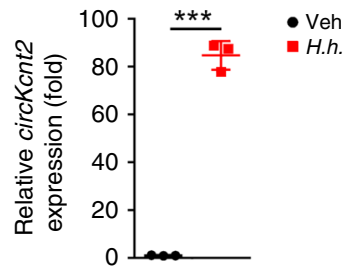

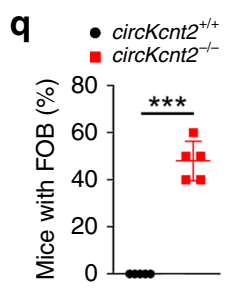

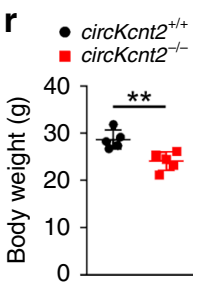

$\mathbf{t}$

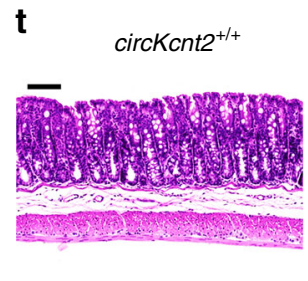

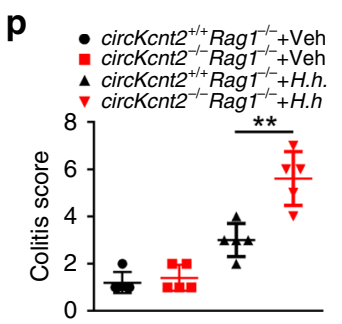

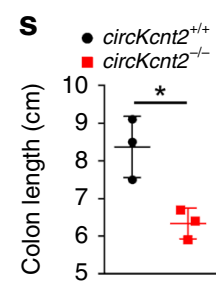

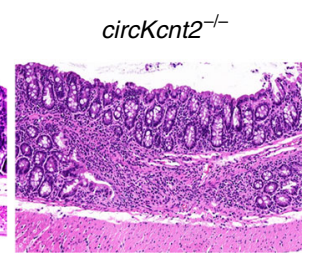
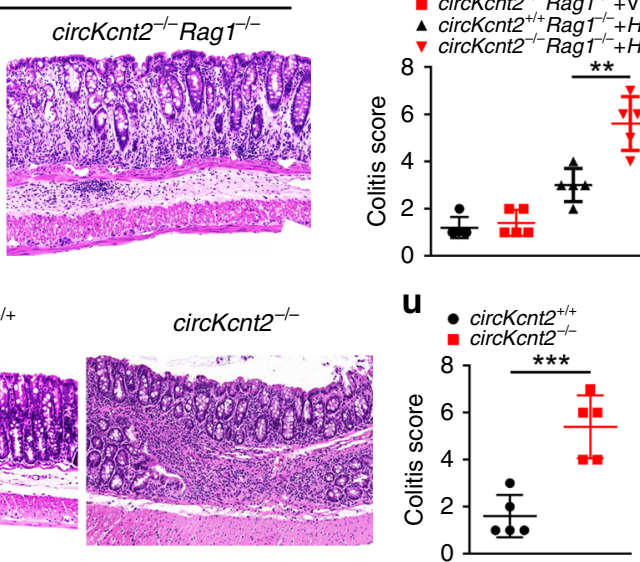

more severe colon injury and more substantial lymphocytes infiltration than $\operatorname{circKcnt} 2^{+/+}$Rag1 $1^{-/-}$mice (Fig. 2f). Furthermore, circKcnt $2^{-1-}$ Rag1 ${ }^{-1-}$ mice exhibited much higher colitis scores and much lower survival rates (Fig. 2g, h). In addition, with adoptive transfer assay, engraftment of circKcnt2-deficient ILC3s with circKcnt2 overexpression could attenuate innate colitis (Supplementary Fig. 3l, m). FITC-dextran by gavage is used to measure mouse intestinal damage ${ }^{8}$ We observed that circKcnt $2^{-1-}$ Rag1 $^{-1-}$ mice exhibited much higher concentrations of blood FITC-dextran (Fig. 2i), suggesting severe intestinal injury of circKcnt $2^{-1-}$ Rag1 $1^{-1-}$ mice. Therefore, circKcnt2 deletion promotes induction of mouse innate colitis.

In addition, IL-17+ ${ }^{+}$ILC3 numbers were gradually increased in circKcnt2 $2^{-/-}$Rag1 $1^{-1-}$ mice with DSS treatment, reaching a peak 
Fig. 2 CircKcnt2 knockout induces ILC3 activation and causes spontaneous colitis. a mRNA levels of indicated genes were tested by qRT-PCR in ILC3s from DSS-treated circKcnt2 $2^{+/+}$Rag1 $^{-/-}$and circKcnt2 ${ }^{-/-}$Rag1-/- mice. b Analysis of IL-17+ ILC3s (Lin ${ }^{-}$CD45 ${ }^{+}$ROR Rt $^{+}$gated) in DSS-treated circKcnt2 ${ }^{+/+}$Rag1 $^{-/-}$ and circKcnt2 ${ }^{-/-}$Rag1 ${ }^{-/}-$mice $\left.{ }^{* \star \star} P<0.0001\right)$. Numbers of total and IL-17+ ILC3s were calculated in the right panels. $n=5$ for each group. $\mathbf{c}$ Geometric mean fluorescence intensity (MFI) of IL-17+ ILC3s was shown ( $\left.{ }^{\star \star} P=0.0008\right) . n=3$ independent samples. d ILC3s were isolated from DSS-treated mice and incubated with or without IL-23 for $24 \mathrm{~h}\left({ }^{\star \star} P=0.0041\right)$. Secreted IL-17 was detected by ELISA. Veh vehicle. $n=3$ independent samples. e Body weight changes were calculated. CircKcnt2 ${ }^{+/}+\operatorname{Rag} 1^{-/-}$and circKcnt2-/-Rag1-/- mice were treated with $3 \%$ DSS for 7 days, followed by normal water for 7 days ${ }^{\star \star \star} P=$ 0.0001). $n=5$ for each group. f After DSS treatment, colon sections from circKcnt $2^{+/+}$Rag $^{-/-}$and circKcnt $2^{-/-}$Rag $1^{-/}$mice were analyzed by H\&E staining. Scale bar, $100 \mu \mathrm{m}$. $\mathbf{g}$ Colitis scores of indicated mice were analyzed ( $\left.{ }^{\star \star} P=0.0071\right) . n=5$ for each group. h After DSS treatment, survival rates of circKcnt2 $+/+$ Rag $^{-/-}$and circKcnt2 ${ }^{-/-}$Rag1 ${ }^{-/-}$mice were monitored. $n=10$ for each group. $\mathbf{i}$ CircKcnt2 ${ }^{+/+}$Rag $^{-/-}$and circKcnt2 ${ }^{-/-}$Rag1 ${ }^{-/-}$mice treated with $3 \%$ DSS for 5 days were administrated with FITC-dextran by gavage. Translocation of FITC-dextran into mouse serum was detected ( $\left.{ }^{\star \star \star} P=0.0005\right)$. $n=5$ independent

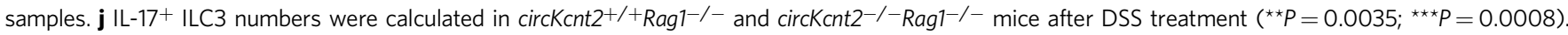
$n=5$ for each group. $\mathbf{k} \mathrm{H} \& E$ staining of colons from indicated mice treated with 3\% DSS and administrated with anti-IL-17 or isotype IgG. Scale bar, $100 \mu$ m.

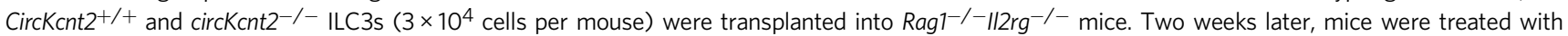
DSS. I Colitis scores of indicated mice treated as in $\mathbf{k}$ were analyzed $\left({ }^{\star \star} P=0.0033\right) . n=5$ for each group. $\mathbf{m}$ Body weight changes of mice in $\mathbf{k}$ were calculated $\left.{ }^{\star \star \star} P=0.0005\right) . n=5$ for each group. $\mathbf{n}$ CircKcnt2 expression was tested by qRT-PCR in ILC3s from H.h.-infected $I 110^{-} /-$mice $\left({ }^{\star \star \star} P<0.0001\right) . n=3$ independent samples. o After H.h. infection, colon sections from circKcnt $2^{+/+}$Rag $1^{-/-}$and circKcnt2-/-Rag1-/- mice were analyzed by H\&E staining. Scale bar,

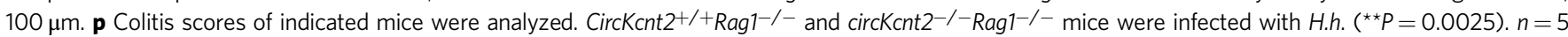
independent samples. q Frequencies of fecal occult blood (FOB) in circKcnt2 ${ }^{+/+}$and circKcnt2 $2^{-/-}$mice were analyzed $\left({ }^{\star \star *} P<0.0001\right)$. $n=5$ independent samples. $\mathbf{r}$ Body weights of circKcnt2 ${ }^{+/+}$and circKcnt2 ${ }^{-/-}$mice were measured $\left.{ }^{\star \star} P=0.0065\right) . n=5$ independent samples. $\mathbf{s}$ Colon lengths of circKcnt2 ${ }^{+/+}$ and circKcnt2 ${ }^{-/-}$mice were detected $\left({ }^{\star} P=0.0176\right) . n=3$ independent samples. $\mathbf{t}$ H\&E staining of colon sections from circKcnt $2^{+/+}$and circKcnt2 ${ }^{-/-}$mice. Scale bar $100 \mu \mathrm{m}$. u Colitis scores of circKcnt2 ${ }^{+/+}$and circKcnt2-/- mice $\left({ }^{\star \star} P=0.0007\right) . n=5$ for each group. ${ }^{\star} P<0.05,{ }^{\star \star} P<0.01$ and ${ }^{\star \star \star} P<0.001$. Data were analyzed by an unpaired Student's $t$-test and shown as means \pm SD. Data are representative of at least three independent experiments. Source data are provided as a Source Data file.

number at day 9 in line with a peak level of innate inflammation (Fig. 2j). We next isolated circKcnt2 $2^{+/+}$and $\operatorname{circKcnt2^{-1-}}$ ILC3s $^{-}$ and transplanted them into Rag1-l- $\mathrm{Il2rg}^{-1-}$ mice to test their roles in the regulation of innate inflammation. Two weeks later, recipient mice were administrated with anti-IL-17 antibody or isotype IgG, followed by DSS treatment. We noticed that recipient mice with $\operatorname{circKcnt2^{-1}}$ ILC3 transplantation showed much more severe colon injury and lymphocytes infiltration (Fig. 2k), and consequently exhibited higher colitis scores and more severe body weight loss (Fig. 2l, m). However, anti-IL-17 antibody treatment attenuated innate colitis scores and body weight loss of Rag1 ${ }^{-1-}$ Il $2 \mathrm{rg}^{-1-}$ mice (Fig. $2 \mathrm{k}-\mathrm{m}$ ). To exclude the effect of Kcnt2 on innate colitis induction, we established Kcnt2 KO mice via CRISPR/Cas9 technology (Supplementary Fig. 4a). We found that Kcnt2 deletion did not affect circKcnt2 expression (Supplementary Fig. 4b). We next generated Kcnt2 $2^{-1-}$ Rag $1^{-1-}$ mice through crossing $\mathrm{Kcnt}^{-1-}$ mice with Rag1 ${ }^{+/}$mice, followed by DSS treatment. We observed that Kont2 $2^{-I}$ Rag1 ${ }^{-1-}$ mice did not affect lymphocytes infiltration, colon injury, body weight loss and colitis scores compared with Kcnt2 ${ }^{+/+}$Rag1-l- mice (Supplementary Fig. $4 c-e)$, suggesting Kcnt2 has no influence on innate colitis induction.

Helicobacter hepaticus (H.h.)-induced chronic colitis is IL-17 dependent ${ }^{9}$. H.h. infection can cause colitis in $I l 10^{-/-}$mice $^{31}$. We first determined whether circKcnt2 expression was also induced by $H . h$. infection. $I l 10^{-1-}$ mice were infected with $H . h$., followed by detection of circKcnt2 expression in ILC3s. We noticed that circKcnt2 was markedly upregulated in ILC3s from H.h. infected $I l 10^{-/-}$mice (Fig. 2n). We then infected circKcnt $2^{+/}$Rag1 $^{-/-}$ and circKcnt2 $2^{-1-}$ Rag1 $1^{-1-}$ mice with H.h. to confirm the role of circKcnt2 in IL-17-induced chronic colitis. We found that circKcnt $2^{-I-} \mathrm{Rag1}^{-/-}$mice displayed more substantial infiltration of inflammatory cells and higher colitis scores after 8 week challenge than circKcnt2 ${ }^{+/+} \mathrm{Rag1}^{-1-}$ mice (Fig. 2o, p). Intestinal colonization of H.h. was comparable between circKcnt2 $2^{+/+}$ Rag1 $^{-1-}$ and circKcnt $2^{-I-}$ Rag1 $^{-I-}$ mice (Supplementary Fig. 4f), suggesting that this colitis discrepancy was not due to increased intestinal colonization by H.h. More importantly, circKcnt $2^{-1-}$ mice displayed rectal bleeding, lower body weight and shorter colon length at about 8 months of age (Fig. 2q-s), as well as more infiltration of inflammatory cells and higher colitis scores (Fig. 2t, u), indicating $\operatorname{circKcnt2^{-/-}}$ mice underwent spontaneous colitis. Collectively, circKcnt2 knockout induces ILC3 activation and promotes innate intestinal colitis.

CircKcnt2 suppresses Batf transcription. To explore the molecular mechanism of circKcnt2 in the regulation of ILC3 activity, we isolated circKcnt $2^{+/+}$and circKcnt2 ${ }^{-1-}$ ILC3s from DSStreated mice and performed transcriptome microarray analysis. We found that circKcnt2 deletion caused upregulation of many TFs (Fig. 3a). Among top ten upregulated TFs, Batf was the most upregulated (Fig. 3a, b). Batf upregulation of circKcnt2 $2^{-1-}$ ILC3s was also confirmed by western blot (Fig. 3c). Through chromatin isolation by RNA purification (CHIRP) assay, we observed that circKcnt2 was enriched on the promoter region $(-1800$ to -1600 ) of Batf (Fig. 3d). We found that circKcnt2 bound to the segment ( -1800 to -1600$)$ of Batf promoter via hybridization with circKcnt2 linearized RNAs (Fig. 3e). This result was further validated by luciferase assay (Fig. 3f). These data suggest that circKcnt2 binds Batf promoter to regulate its transcription.

CircRNA loops have critical roles in the RNA interactome ${ }^{23}$. We predicted the loop structure of circKcnt2 with bioinformatics tool (Supplementary Fig. 4g). To determine which loop (HR1, HR2, HR3, HR4, and HR5) was required for the association between circKcnt2 and Batf promoter, we constructed circKcnt2 mutations to abrogate loop structures (Supplementary Table 1). We found that HR4 was required for their interaction (Fig. 3g). Through sequence alignment, we noticed that a pairing complementary bases existed between Batf promoter ( -1800 to -1600 ) and HR4 of circKcnt2 (Fig. 3h). We generated mutations of Batf promoter and circKcnt2 transcript to abrogate their binding, followed by hybridization assay. We found that the binding base mutations really abolished their interactions (Fig. 3h). Through ChIP assay, we found that circKcnt2 deletion promoted enrichment of $\mathrm{H} 3 \mathrm{~K} 27 \mathrm{ac}$ on Batf promoter in ILC3s (Fig. 3i). By contrast, circKcnt2 deletion decreased H3K27me3 enrichment on Batf promoter in ILC3s (Fig. 3j). To assess density of nucleosomes in Batf promoter, we performed DNaseI accessibility assay. As shown, Batf promoter in circKcnt2 $2^{-1-}$ ILC3s was more susceptible to DNaseI digestion (Fig. 3k). 
a

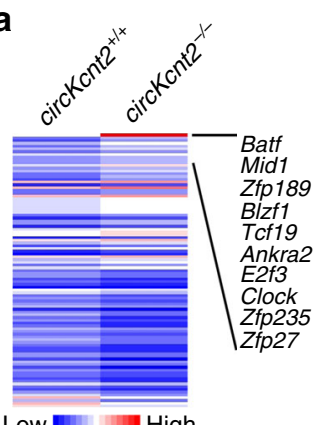

Low b

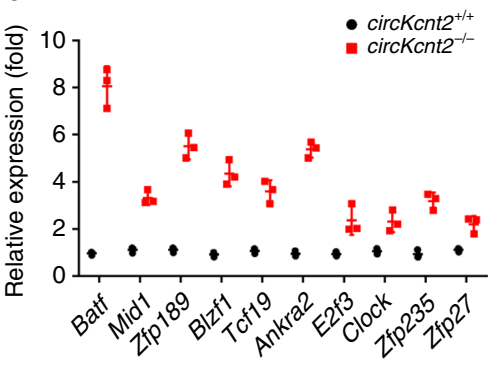

C

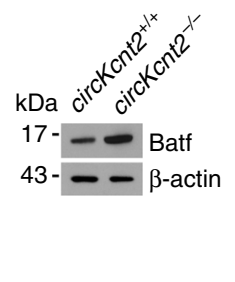

d

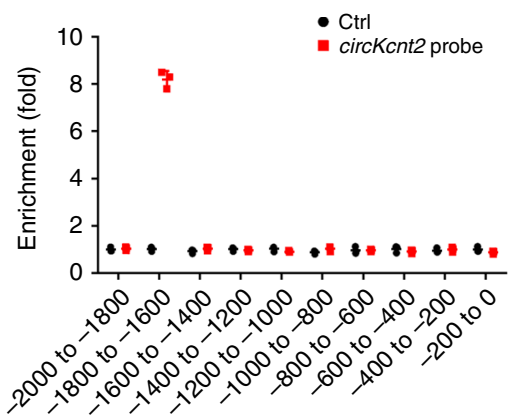

e
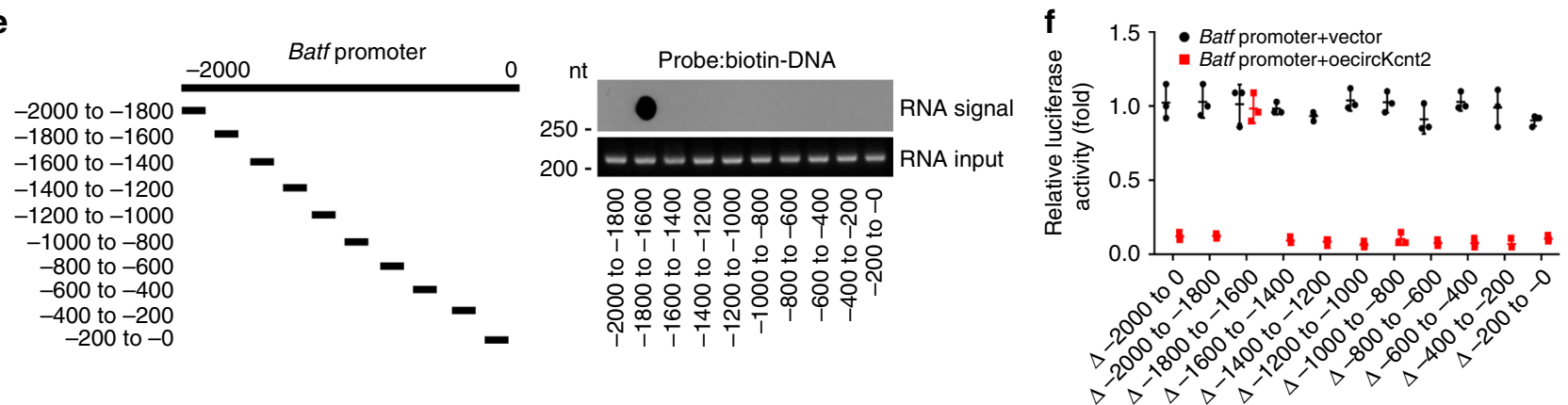

g

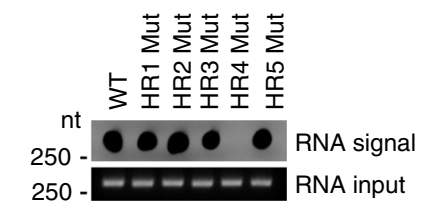

h Batf promoter: 5'-GGGCTCTCTGGT-3' | | | I | | $\vdots$ | | I circKcnt2: 3 '-ACCGAGAUACCU-5' Batf Pro-Mut: - - + + Batf Pro-WT: ++-circKcnt2-Mut: -+-+ circKcnt2-WT: + - + -

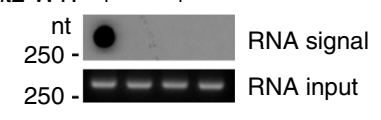

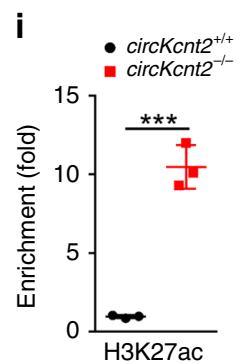

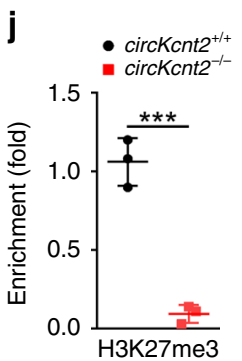

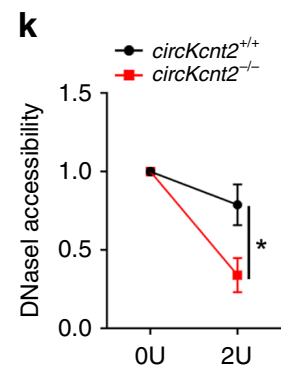

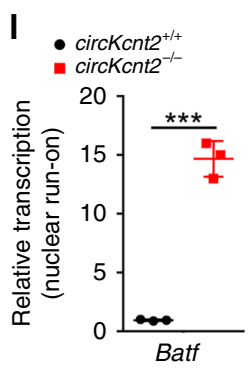

m - Ctrl

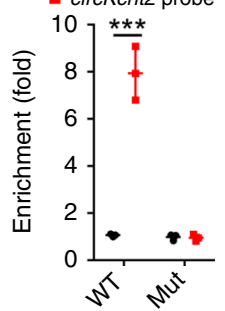

n

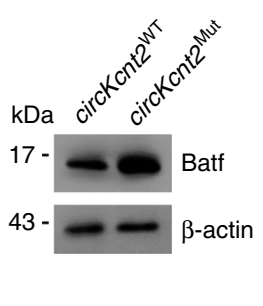

o
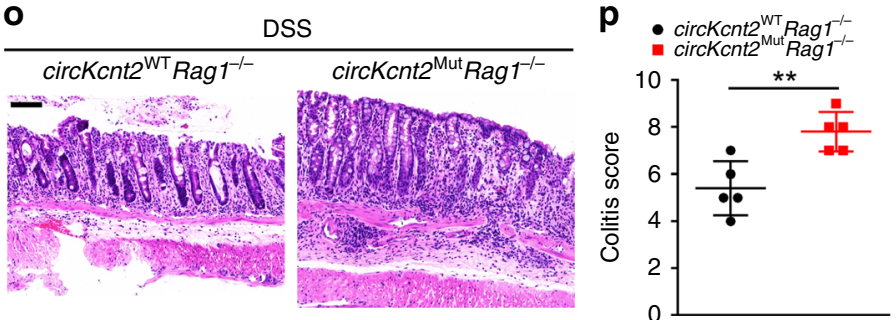

q

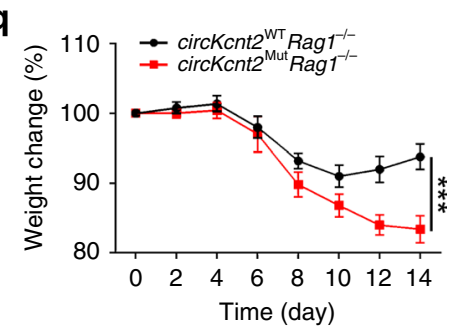

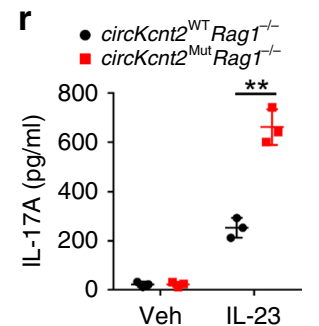

S

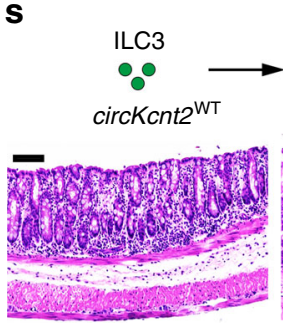

t

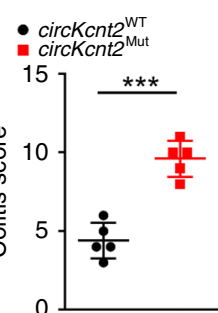

Consistently, circKcnt2 deficiency caused elevated transcription of Batf mRNA by nuclear run-on assay (Fig. 31). These data indicate that circKcnt2 interacts with Batf promoter to suppress its transcription.

To further confirm the in vivo role of their pairing bases, we generated circKcnt2-mutant (called $\operatorname{circKcnt} 2^{\mathrm{Mut}}$ ) mice through knocking in the mutation bases of pairing circKcnt2 region
(Supplementary Fig. 4h). We observed that circKcnt2 mutation abrogated the enrichment of circKcnt2 onto Batf promoter (Fig. $3 \mathrm{~m}$ ), and consequently enhanced Batf expression in ILC3s with DSS treatment (Fig. 3n). We then treated circKcnt2WT Rag1 $1^{-1-}$ and circKcnt $2^{\text {Mut }}$ Rag1 $1^{-1-}$ mice with DSS. We noticed that $\operatorname{circKcnt2} 2^{\mathrm{Mut}} \mathrm{Rag} 1^{-1-}$ mice displayed much more severe innate colitis compared to $\operatorname{circKcnt} 2^{\mathrm{WT}} \operatorname{Rag}^{-1-}$ mice (Fig. 3o-q). 
Fig. 3 CircKcnt2 suppresses Batf transcription. a Heatmap analysis according to differentially expressed TFs in circKcnt2 $2^{+/+}$and circKcnt2 ${ }^{-/-}$ILC3s isolated from DSS-treated mice. b ILC3s were isolated from circKcnt2 $2^{+/+}$and circKcnt $2^{-/-}$mice after DSS treatment, followed by RNA extraction and qRTPCR analysis for top ten upregulated TFs. $n=3$ independent samples. c Batf expression was tested by western blotting in circKcnt2 $+/+$ and circKcnt2-/ILC3s isolated from DSS-treated mice. d ILC3s from DSS-treated mice were lyzed and subjected to CHIRP assay using biotin-labeled probes against circKcnt2. Precipitated DNA fragments were extracted and enrichment of circKcnt2 on Batf promoter was analyzed by PCR. $n=3$ independent samples. e CircKent2 linearized RNA was immobilized on NC membranes, followed by probing with indicated biotin-labeled DNA probes. $\mathbf{f}$ Luciferase reporter assay using Batf truncated promoters with overexpression of circKcnt2 or vector control. $n=3$ independent samples. $\mathbf{g}$ CircKcnt2 linearized RNAs were immobilized onto membrane, followed by probing with biotin-labeled single-strand DNA. HR hairpin region. $\mathbf{h}$ A base pairing between Batf promoter $(-1800$ to -1600$)$ and circKent2 HR4 was predicted. WT or mutant circKent2 HR4 RNAs were immobilized onto membrane, followed by probing with WT or mutant DNA probes. $\mathbf{i}, \mathbf{j}$ ChIP assay was performed using anti-H3K27ac or anti-H3K27me3 with circKcnt2 $+/+$ and circKcnt2 ${ }^{-/-}$ILC3 cell lysates after DSS treatment $\left.{ }^{\star \star \star} P=0.0003 ;{ }^{\star \star \star} P=0.0006\right) . n=3$ independent samples. $\mathbf{k}$ DNasel accessibility assay was conducted using circKcnt2 ${ }^{+/+}$and circKcnt2 ${ }^{-/}$ILC3s from DSS-treated mice $\left({ }^{\star} P=0.0102\right) . n=3$ independent samples. I CircKcnt $2^{+/+}$and circKcnt2 ${ }^{-/-}$ILC3s were subjected to nuclear run-on assay, followed by detection of Batf transcription by RT-PCR analysis ( $\left.{ }^{\star \star \star} P=0.0001\right) . n=3$ independent samples. $\mathbf{m}$ CircKcnt2 enrichment on Batf promoter was tested by CHIRP assay using circKcnt2WT and circKcnt2Mut ILC3 lysates after DSS treatment $\left({ }^{\star \star \star} P=0.0005\right)$. $n=3$ independent samples. n Batf expression was measured using western blotting in circKcnt2WT and circKcnt2Mut ILC3s after DSS treatment. o H\&E staining of colon tissues from

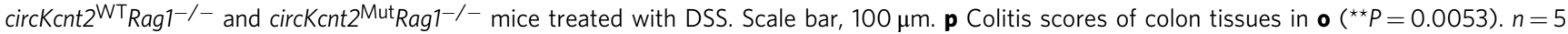
independent samples. q CircKcnt2 ${ }^{\mathrm{WT}} \mathrm{Rag}^{-1-}$ and circKcnt2 ${ }^{\mathrm{Mut}} \mathrm{Rag}^{-1-}$ mice were treated with DSS and weight changes were monitored ( $\left.{ }^{\star \star \star} P<0.0001\right)$.

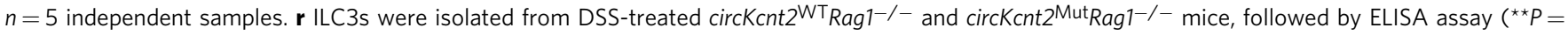
0.0011). Veh vehicle. $n=3$ independent samples. s ILC3s isolated from circKcnt2WT and circKcnt2Mut mice were transplanted into Rag1-/-II2rg-/- mice. Two weeks later, recipient mice were administrated with DSS. Colons were isolated and analyzed by H\&E staining. Scale bar, $100 \mu \mathrm{m}$. $\mathbf{t}$ Colitis scores of indicated mice treated as in $\mathbf{s}$ were analyzed ( ${ }^{\star \star \star} P<0.0001$ ). $n=5$ for each group. ${ }^{\star} P<0.05,{ }^{\star \star} P<0.01$, and ${ }^{\star \star \star} P<0.001$. Data were analyzed by an unpaired Student's $t$-test and shown as means \pm SD. Data are representative of at least three independent experiments. Source data are provided as a Source Data file.

In addition, $\operatorname{circKcnt} 2^{\mathrm{Mut}} \mathrm{Rag} 1^{-1-}$ ILC3s produced more IL-17 in vitro (Fig. 3r). We also noticed that $\operatorname{circKcnt} 2^{\mathrm{Mut}} \mathrm{Rag} 1^{-1-}$ mice did not affect ILC3 development (Supplementary Fig. 4i). circKcnt $2^{\text {Mut }}$ ILC3s did not undergo apparent cell death after DSS treatment (Supplementary Fig. 4j). Finally, we isolated ILC3s from $\operatorname{circKcnt} 2^{\mathrm{Mut}}$ and $\operatorname{circKcnt} 2^{\mathrm{WT}}$ mice, and transplanted them into $\mathrm{Ragl}^{-1} \mathrm{Il2} \mathrm{rg}^{-1-}$ mice with DSS treatment. We found that mice engrafted with $c i r c K c n t 2^{\text {Mut }}$ ILC3s exhibited severe innate colitis (Fig. 3s, t). Altogether, circKcnt2 enriches onto Batf promoter to inhibit its expression leading to suppression of ILC3 activation.

CircKcnt2 associates with Mbd3. To further reveal how circKcnt2 suppressed Batf transcription in ILC3s, we conducted RNA pulldown assay using biotin-labeled linearized circKcnt2 as bait, followed by silver staining and mass spectrometry. We found that circKcnt2 bound to Mbd3 from gut lamina propria lymphocyte (LPL) lysates (Fig. 4a, Supplementary Fig. 5a). Mbd3 is a major component of the NuRD complex ${ }^{32}$. We noticed that circKcnt2 could precipitate the main components of the NuRD complex in LPL lysates (Fig. 4b). The direct interaction between circKcnt2 and Mbd3 was further validated by EMSA assay (Fig. 4c). Moreover, only Mbd3 of the NuRD complex components directly interacted with circKcnt2 (Fig. 4d). The interaction between circKcnt2 and Mbd3 in LPL lysates was further confirmed via RNA pulldown assay using biotin-labeled circKcnt2 (Fig. 4e). Consistently, circKcnt2 was co-localized with Mbd3 in ILC3s (Fig. 4f). In addition, circKcnt2 was enriched by Mbd3 by RNA immunoprecipitation assay (Fig. 4g).

To further determine which loop of circKcnt2 transcript was required for the interaction between circKcnt2 and Mbd3, we performed RNA pulldown assay using biotin-labeled mutant circKcnt2. We observed that HR5 mutation abolished their association of circKcnt2 with Mbd3 (Fig. 4h). This result was further validated by EMSA assay (Fig. 4i). Collectively, circKcnt2 directly interacts with Mbd3.

CircKcnt 2 recruits the NuRD complex onto Batf promoter. We next sought to examine whether circKcnt2 could recruit the
NuRD complex onto Batf promoter to regulate its transcription. Through ChIP assay, we found that Mbd3 was enriched on the same region of Batf promoter as circKcnt2 (Fig. 5a). However, circKcnt2 deletion abrogated enrichment of the NuRD complex components on Batf promoter (Fig. 5b). Interestingly, the NuRD complex could not enrich on Batf promoter in circKcnt $2^{\mathrm{Mut}}$ ILC3s (Fig. 5c). Of note, Mbd3 co-localized with Batf promoter in WT ILC3s but not in circKcnt2-1- ILC3s (Fig. 5d). Moreover, with cross-linking treatment, the NuRD complex was co-eluted with Batf promoter in circKcnt2 $2^{+/+}$LPL lysates, but not in circKcnt $2^{-l-}$ LPL lysates (Fig. 5e). Through CHIRP assay, the NuRD complex and Batf promoter were co-eluted in $c i r c K c n t 2 \mathrm{WT}$ LPL lysates, but not in circKcnt $2^{\text {Mut }}$ LPL lysates (Fig. 5f). These data indicate that circKcnt2 recruits the NuRD complex onto Batf promoter in ILC3s.

To further test whether the NuRD complex regulated Batf

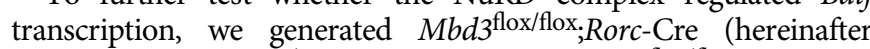
referred to as $M b d 3^{-/-}$) mice via crossing $M b d 3^{\text {flox/flox }}$ mice with Rorc-Cre mice. Mbd3 was completely deleted in $M b d 3^{-I-}$ mice (Supplementary Fig. 5b). We also generated $M b d 3^{-1-}$ circKcnt $2^{-1-}$ mice through crossing $M b d 3^{-1-}$ mice with circKcnt $2^{-1-}$ mice. We found that Batf promoter enriched substantial H3K27ac in $M b d 3^{-1-}$ and $\mathrm{Mbd3}^{-1-}$ circKcnt2-/- ILC3s (Fig. 5g). In addition, Batf promoter in $\mathrm{Mbd3}^{-1-}$ and Mbd3 $3^{-l-}$ circKcnt2 ${ }^{-I-}$ ILC3s was more susceptible to DNaseI digestion compared to that of WT ILC3s (Fig. 5h). Consistently, Batf mRNA was more actively transcribed in $\mathrm{Mbd3}^{-1-}$ and $M b d 3^{-1-}$ circKcnt2 $2^{-1-}$ ILC3s by nuclear run-on assay (Fig. 5i). Consequently, Batf was highly expressed in $\mathrm{Mbd3}^{-{ }^{-}}$and Mbd3 ${ }^{-I-}$ circKcnt2 $2^{-I-}$ ILC3s (Fig. 5j, k). Taken together, circKcnt2 recruits the NuRD complex to inhibit Batf transcription.

Batf triggers ILC3 activation and colitis induction. To further examine the role of Batf in the regulation of ILC3s, we generated Batf knockout (KO) mice (Supplementary Fig. 5c). We then established Batf ${ }^{-1-}$ Rag1 $^{-1-}$ mice via crossing Batf ${ }^{-1-}$ mice with Rag1 ${ }^{+/}$mice, followed by DSS treatment for colitis induction. We observed that Batf deletion decreased numbers of $\mathrm{IL}-17^{+}$ILC3s during intestinal inflammation (Fig. 6a). Moreover, Batf $f^{-/-}$ Rag1 ${ }^{-1-}$ ILC3s secreted less amounts of IL-17 (Fig. 6b, c). It has 
a
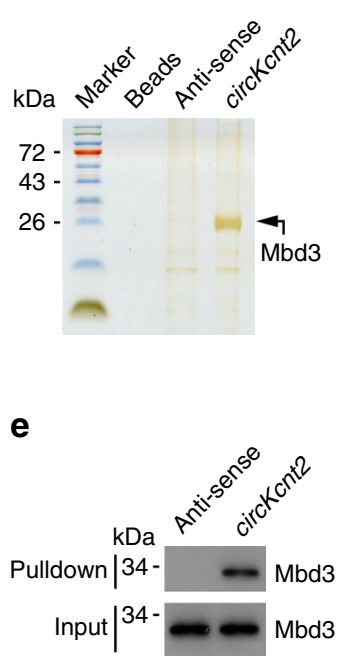

b

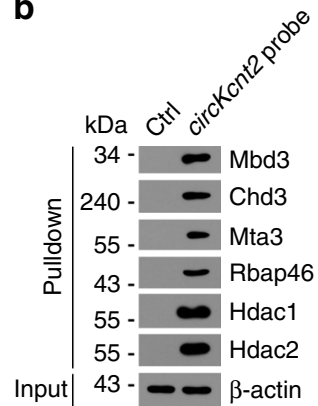

C

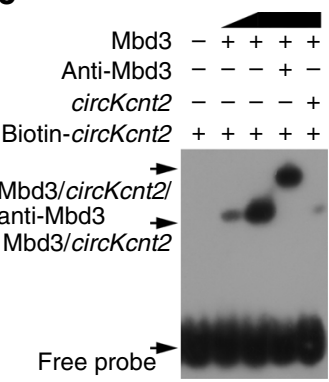

d

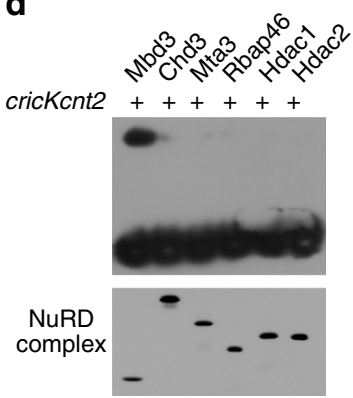

f
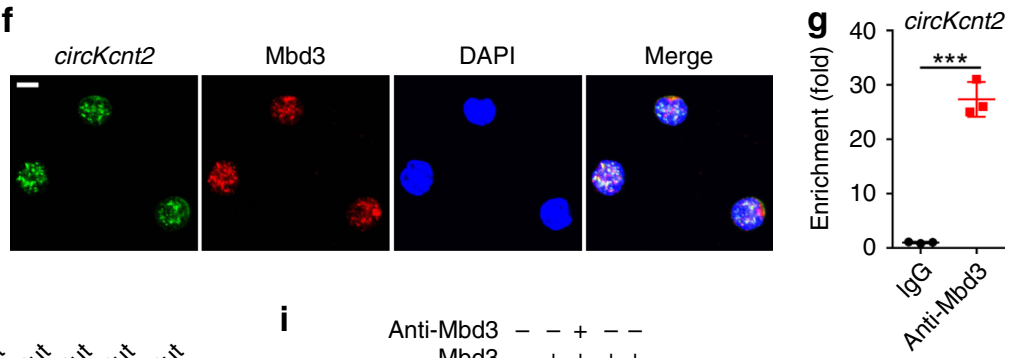

h

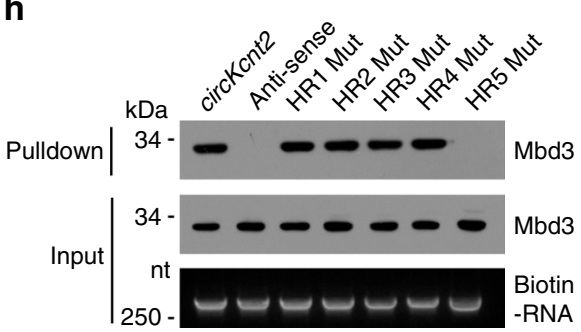

Fig. 4 CircKcnt2 associates with Mbd3. a LPL cells from DSS-treated WT mice were lysed and incubated with biotin-labeled and linearized circKcnt2 transcripts, anti-sense, or Sepharose 4B beads control. Precipitants were resolved by SDS-PAGE, followed by silver staining. Indicated bands were identified via mass spectrometry. b LPL lysates were incubated with circKcnt2 probes or controls, followed by western blotting using indicated antibodies. c Electrophoretic mobility shift assay (EMSA) using biotin-labeled circKcnt2 and recombinant Mbd3 with or without anti-Mbd3. d EMSA assay using linearized circKcnt2 RNAs and Flag tagged NuRD complex component proteins. e RNA pulldown assay was performed using linearized and biotin-labeled circKcnt2 RNAs or anti-sense through LPL cell lysates. $\mathbf{f}$ CircKcnt2 was co-localized with Mbd3 in ILC3s by immunofluorescence staining. Scale bar, $5 \mu$ m. g RNA immunoprecipitation (RIP) assay was conducted using anti-Mbd3 or lgG through LPL lysates from DSS-treated mice ( $\left.{ }^{\star \star \star} P=0.0001\right) . n=3$ independent samples. h RNA pulldown assay was performed using WT circKcnt2 and its different mutations. i EMSA assay using WT circKcnt2 or HR5 mutation. ${ }^{\star \star \star} P<0.001$. Data were analyzed by an unpaired Student's $t$-test and shown as means $\pm S D$. Data are representative of at least three independent experiments. Source data are provided as a Source Data file.

been reported that Batf can induce $I l 17 a$ transcription in $\mathrm{T}$ cells $\mathrm{s}^{33}$. We next sought to test whether Batf could initiate Il17a transcription in ILC3s. We found that Batf enriched on Ill7a promoter in ILC3s (Fig. 6d). We noticed that Batf and Il17a promoter colocalized in ILC3s by fluorescence in situ hybridization assay (Fig. 6e). The direct interaction between Batf and Il17a promoter in ILC 3s was further validated by EMSA (Fig. 6f). In addition, Batf promoted Il17a transcription in WT ILC3s via luciferase reporter assay (Fig. 6g). Of note, Batf deletion dramatically suppressed Il17a transcription (Fig. 6h, i). More importantly, Batf ${ }^{-1}$ Rag1 $^{-1-}$ mice exhibited mild intestinal inflammation and slight injury (Fig. 6j-l). Altogether, Batf initiates Il17a expression to induce ILC3 activation leading to colitis induction.

Batf deletion promotes colitis resolution. To further determine the physiological role of Mbd3 in the modulation of ILC3s and intestinal innate colitis, we generated $M b d 3^{-1-}$ Rag $1^{-1-}$ mice. We observed that $M b d 3^{-1-}$ Rag1 $1^{-1-}$ ILC3s produced more substantial amounts of IL-17 after DSS challenge, similar to circKcnt2 $2^{-1-}$ Rag1 ${ }^{-/-}$ILC3s (Fig. 7a). Moreover, numbers of IL-17 ${ }^{+}$ILC3s were

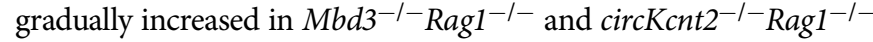
mice during intestinal inflammation (Fig. 7b). We also established circKcnt2 $2^{-1-}$ Batf $^{-1-}$ Rag1 $^{-1-}$ (TKO) mice. We noticed that TKO ILC3s produced much less amounts of IL-17 and TKO mice had lower numbers of IL-17 ${ }^{+}$ILC3s with DSS treatment than $\mathrm{Mbd3}^{-1-} \mathrm{Rag1}^{-1-}$ or circKcnt2 $2^{-1-} \mathrm{Rag1}^{-1-}$ control mice (Fig. 7a, b). Consistently, $M b d 3^{-I-} \operatorname{Rag}^{-I_{-}}$and circKcnt2 ${ }^{-I-}$ Rag1 $^{-1-}$ mice displayed more severe lymphocyte infiltration and intestinal injury, whereas TKO mice exhibited opposite phenotypes (Fig. 7c-e). These data suggest that Mbd3 suppresses ILC3 activation that is opposite to Batf function.

We then transplanted $M b d 3^{-1-}$, circKcnt $2^{-1-}$, circKcnt2 $2^{-1-}$ Batf $^{-1}$ (hereinafter referred to as DKO) or WT ILC3s into Rag1 ${ }^{-1-} I l 2 \mathrm{rg}^{-1-}$ mice, followed by DSS treatment with or without administration of anti-IL-17 antibody. We observed that adoptive transfer of $\mathrm{Mbd3}^{-1-}$ and circKcnt2 $2^{-1-}$ ILC3s caused more severe innate colitis compared to WT ILC3s (Fig. 7f, g). By contrast, engraftment of DKO ILC3s exhibited slighter innate colitis than transfer of $\mathrm{Mbd3}^{-1-}$ or circKcnt2 $2^{-1-}$ ILC3s alone (Fig. 7g, g). Of note, with anti-IL-17 antibody treatment, adoptive transfer of $M b d 3^{-1-}$ and $\operatorname{circKcnt} 2^{-1-}$ ILC3s induced attenuated innate colitis 
a

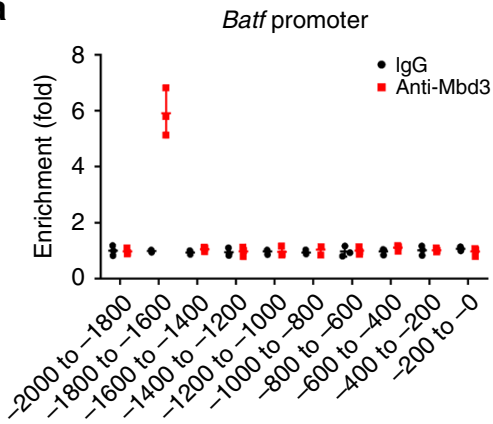

b

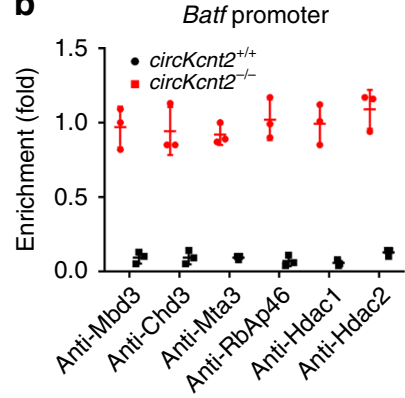

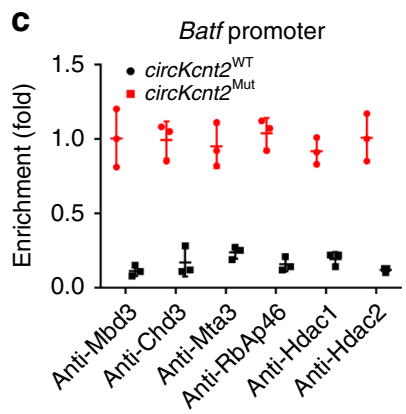

d

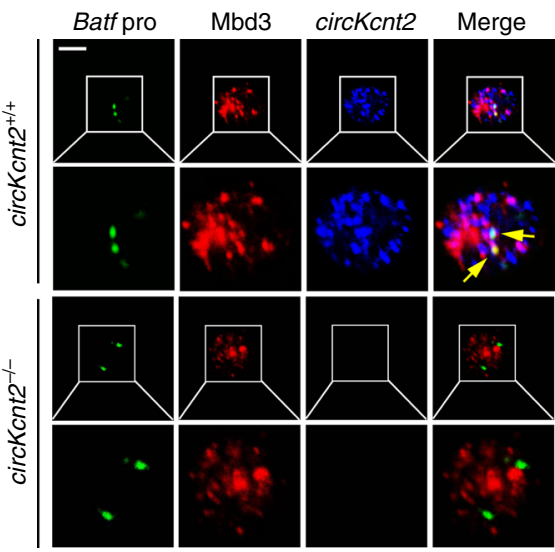

f

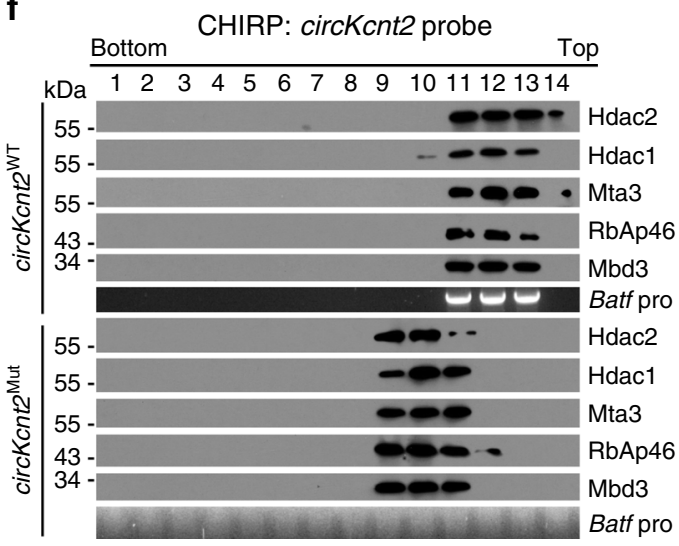

e
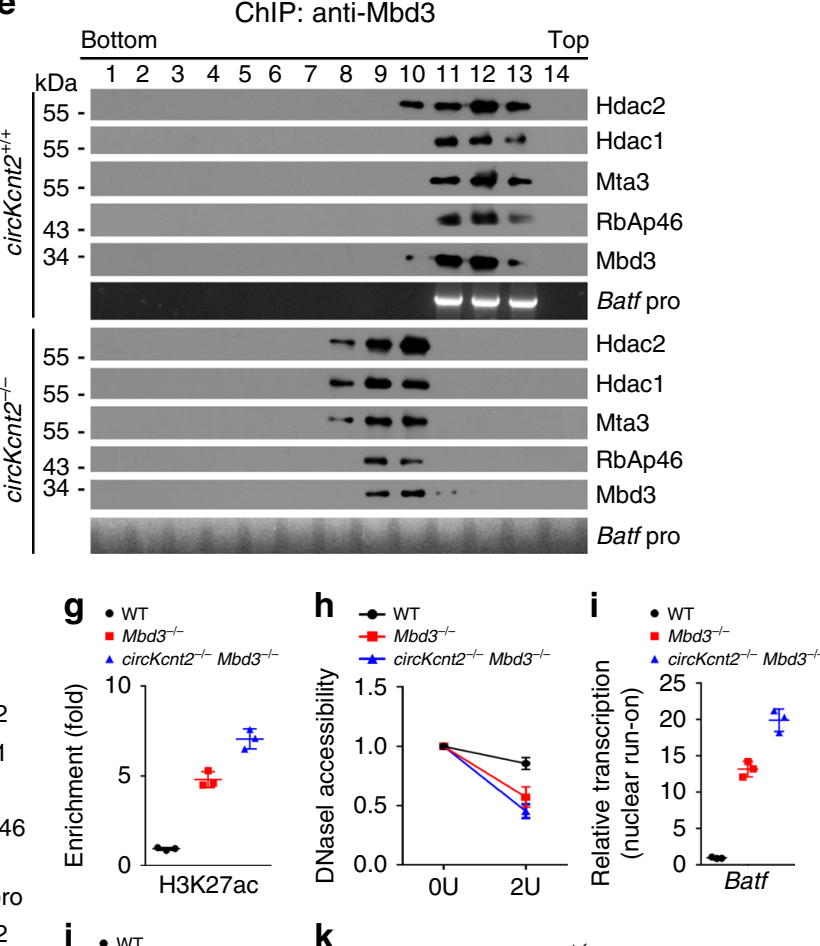

$\mathbf{k}$

- $\mathrm{Mbd3}^{-1-}$

$\triangle$ circk
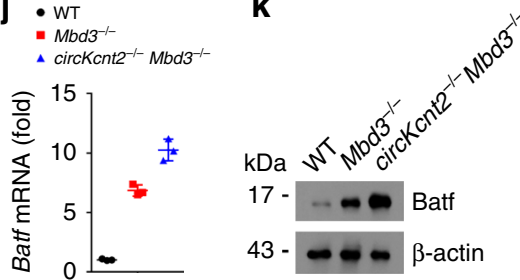

Fig. 5 CircKcnt2 recruits the NuRD complex onto Batf promoter to suppress its expression. a Analysis of Mbd3 enrichment on Batf promoter by ChIP assay. ILC3s was isolated from DSS-treated WT mice. $n=3$ independent samples. $\mathbf{b}$ Enrichment analysis of NuRD complex component proteins on Batf promoter in circKcnt2 ${ }^{+/+}$and circKcnt2 $/-$ILC3s after DSS treatment. $n=3$ independent samples. c ChIP assay for enrichment of NuRD complex component proteins on Batf promoter in circKcnt2WT and circKcnt2Mut ILC3s from DSS-treated mice. $n=3$ independent samples. $\mathbf{d}$ FISH assay for analysis of co-localization between Mbd3 and Batf promoter in circKcnt2 ${ }^{+/+}$and circKcnt2-/- ILC3s from DSS-treated mice. Green, Batf promoter; red, Mbd3; blue, circKcnt2. Scale bar, $5 \mu \mathrm{m}$. e LPLs isolated from DSS-treated circKcnt2 $+/+$ and circKcnt2 ${ }^{-/}-$mice were used for ChIP assay with anti-Mbd3. Eluents were subjected to sucrose gradient ultracentrifugation, followed by western blotting and PCR analysis. $\mathbf{f}$ LPLs were isolated from DSS-treated circKcnt2WT and circKcnt2Mut mice and used for CHIRP assay using biotin-labeled circKcnt2 probes. $\mathbf{g}$ ChIP assay for enrichment analysis of H3K27ac on Batf promoter in $\mathrm{Mbd3}^{-/-}$, circKcnt2 ${ }^{-/-} \mathrm{Mbd3}^{-/-}$, or WT control ILC3s. $n=3$ independent samples. $\mathbf{h}$ DNasel accessibility assay for Batf promoter accessibility in $\mathrm{Mbd3}^{-/-}$, circKcnt2 ${ }^{-/-} \mathrm{Mbd3}^{-/-}$, or WT control ILC3s. $n=3$ independent samples. i ILC3s from DSS-treated Mbd3 ${ }^{-/-}$, circKcnt2 ${ }^{-/-}$Mbd3 ${ }^{-/-}$, or WT control mice were subjected to nuclear run-on assay, followed by RT-PCR analysis of Batf. $n=3$ independent samples. $\mathbf{j}$, $\mathbf{k}$ Analysis of Batf expression by qRT-PCR and western blotting in $\mathrm{Mbd3}^{-/}-$, circKcnt2 ${ }^{-/}-\mathrm{Mbd3}^{-/-}$, or WT control ILC3s. $n=3$ independent samples. Data were analyzed by an unpaired Student's $t$-test and shown as means \pm SD. Data are representative of at least three independent experiments. Source data are provided as a Source Data file. 
a

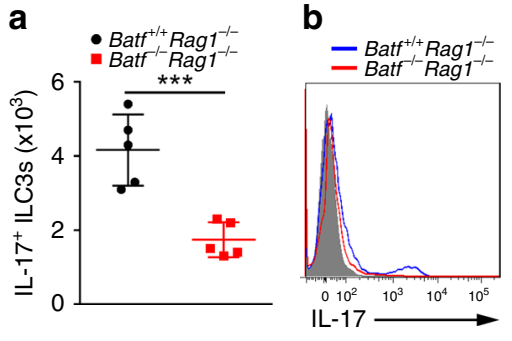

e

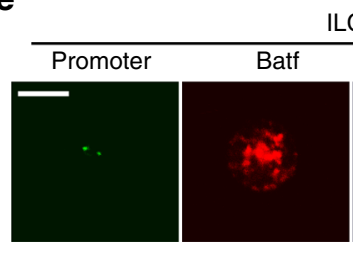

g

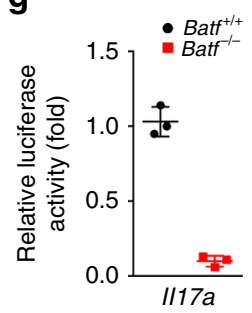

$\mathbf{k}$

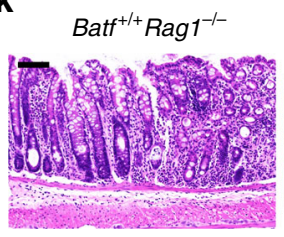

h
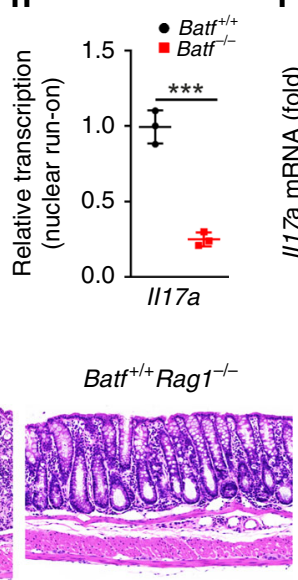

i

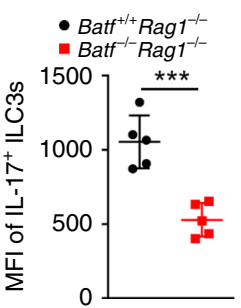

C

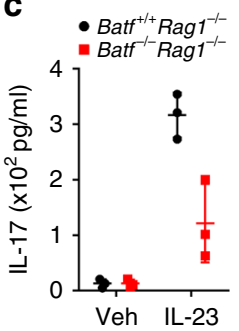

d

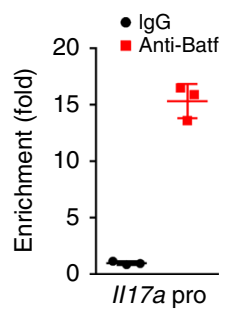

f

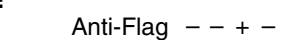

Flag-Batf -+++

Labeled probe ++++

Unlabeled probe ---+
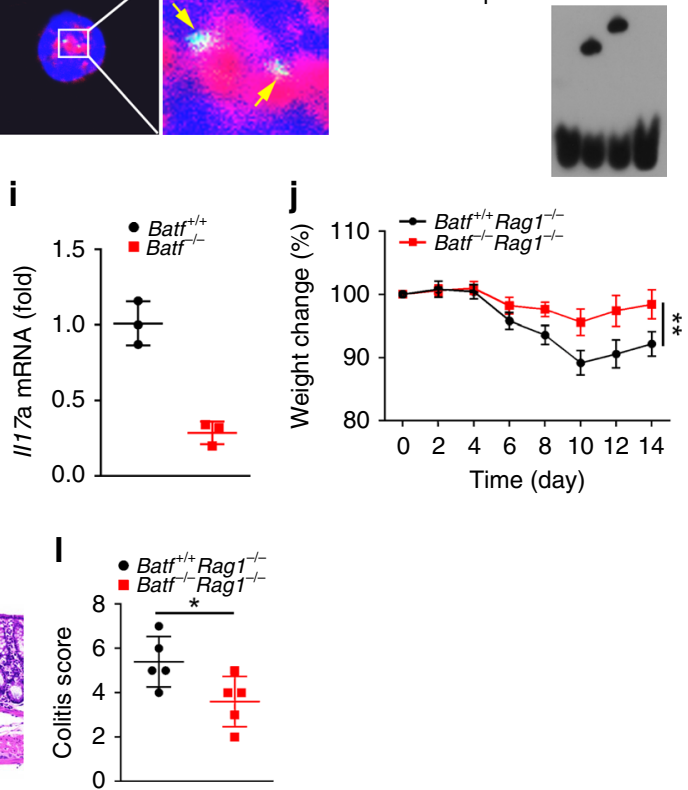

Fig. 6 Batf triggers ILC3 activation and colitis induction. a IL-17+ ILC3 numbers were analyzed in Batf ${ }^{+/}$Rag1 ${ }^{-/-}$or Batf ${ }^{-/-}$Rag1 ${ }^{-/-}$mice after DSS treatment $\left.{ }^{\star \star \star} P=0.0009\right) . n=5$ independent samples. $\mathbf{b}$ Expression of IL-17 was measured in Batf ${ }^{+/+}$Rag $^{-/}{ }^{-}$or Batf ${ }^{-/-}$Rag $1^{-/-}$ILC3s from DSS-treated mice. Geometric MFI of IL-17+ ILC3s was presented in the right panel ( $\left.{ }^{\star \star *} P=0.0005\right) \cdot n=5$ independent samples. c ELISA assay using Batf $+/+$ Rag $1^{-/-}$or Batf ${ }^{-/-}$Rag $^{-1-}$ ILC3s from DSS-treated mice. Veh vehicle. $n=3$ independent samples. d Analysis of Batf enrichment on II17a promoter in ILC3s by ChIP assay. $n=3$ independent samples. e Co-localization between Batf and II17a promoter was analyzed by FISH in ILC3s isolated from DSS-treated WT mice. Green, II17a promoter; red, Batf; blue, nucleus. Scale bar, $5 \mu \mathrm{m}$. $\mathbf{f}$ The interaction between Batf and II17a promoter was tested by EMSA assay. $\mathbf{g}$ Luciferase reporter assay was performed using III7a reporter in Batf ${ }^{+/+}$and Batf ${ }^{-/-}$ILC3s. $n=3$ independent samples. $\mathbf{h}$ Batf H $/+^{+}$and Batf $f^{-/-}$ILC3s were subjected to nuclear run-on assay, followed by RT-PCR analysis of $I 177 a\left({ }^{\star \star \star} P=0.0004\right) . n=3$ independent samples. i Relative expression of II17a in ILC3s isolated from Batf $^{+/+}$and Batf ${ }^{-/}$mice treated with DSS. $n=3$ independent samples. $\mathbf{j}$ Analysis of body weight changes of indicated mice after DSS treatment ${ }^{\star \star \star} P=$ 0.0017). $n=5$ for each group. $\mathbf{k}$ H\&E staining of colons from indicated mice treated with DSS. Scale bar, $100 \mu \mathrm{m}$. I Colitis scores of indicated mice as in $\mathbf{k}\left({ }^{\star \star} P=0.0372\right) . n=5$ independent samples. ${ }^{\star} P<0.05,{ }^{\star} \times P<0.01$, and ${ }^{\star \star \star} P<0.001$. Data were analyzed by an unpaired Student's $t$-test and shown as means \pm SD. Data are representative of at least three independent experiments. Source data are provided as a Source Data file.

(Fig. 7f, g), indicating that IL-17 induced by ILC3s plays a critical role in circKcnt2-mediated inflammatory inhibition. Taken together, circKcnt2 recruits the NuRD complex onto Batf promoter to suppress its expression, which inhibits ILC3 activation to facilitate innate colitis resolution.

\section{Discussion}

ILC3s are an important regulator for immunity, inflammation, and tissue homeostasis in the intestine ${ }^{34}$. Dysregulation of ILC3 activation may lead to intestinal diseases, such as $\mathrm{IBD}^{12}$. However, how ILC3 activation is regulated remains elusive. In this study, we showed that circKcnt2 is highly induced in activated ILC3s during intestinal innate colitis. Deletion of circKcnt2 causes ILC3 activation and severe innate colitis. Mechanistically, circKcnt2 as a nuclear circRNA recruits the NuRD complex onto
Batf promoter to inhibit its expression, which suppresses $I l 17$ expression for ILC3 inactivation to promote innate colitis resolution (Supplementary Fig. 5d). Importantly, with DSS treatment,

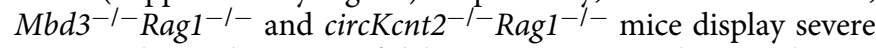
innate colitis, whereas Batf deletion promotes colitis resolution. Therefore, we reveal a circRNA circKcnt2 in regulating ILC3 inactivation and innate colitis resolution.

ILC3s are activated at the early stage of innate immune response that are important for pathogen elimination or tissue repair ${ }^{1}$. Deficiency or dysfunction of ILC3s leads to apparent pathologic disorders $^{35}$. Similar to other immune responses, ILC3 activation also requires to be balanced by stringent regulations. Excessive activation of ILC3s may cause tissue damage and induce inflammatory disorders, such as IBD and tumorigenesis ${ }^{9,36}$. Several extrinsic factors are reported to be involved in the regulation of ILC3 activation. For example, IL-23 and IL-1 $\beta$ directly activates 
a

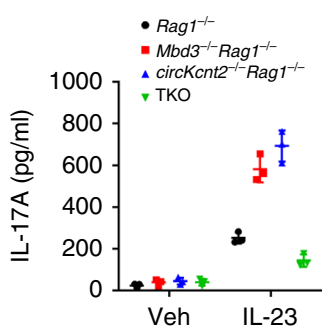

b

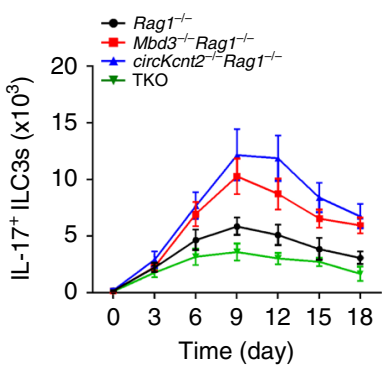

C

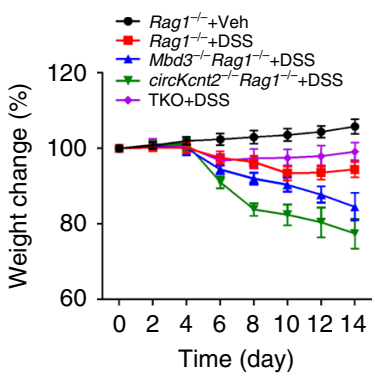

e

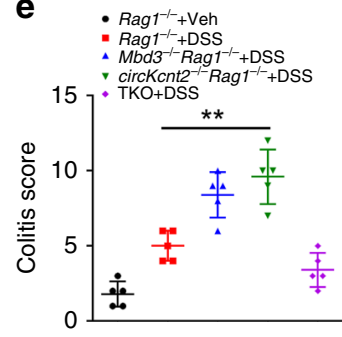

d

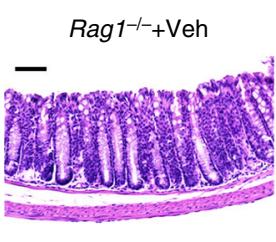

Rag1-1-+DSS
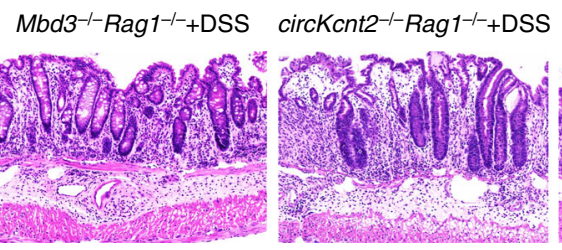

TKO+DSS

f
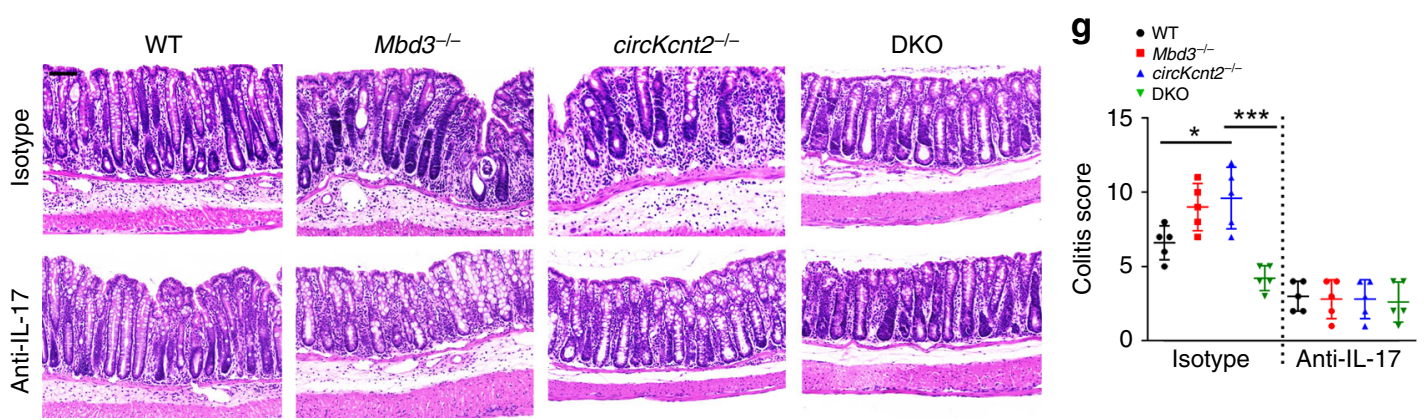

Fig. 7 Mbd3 deletion causes severe innate colitis while Batf deletion promotes colitis resolution. a ILC3s isolated from indicated mice were used for ELISA assay to analyze IL-17 production. TKO, circKcnt2 ${ }^{-/-}$Batf ${ }^{-/-}$Rag $1^{-/-}$mice. $n=3$ independent samples. b Cell numbers of IL-17+ ILC3s were analyzed in indicated mice after DSS treatment. $n=5$ for each group. c Analysis of body weight changes of indicated mice after DSS treatment. $n=5$ for each group. $\mathbf{d}$ H\&E staining of colons from indicated mice treated with DSS. Scale bar, $100 \mu \mathrm{m}$. e Colitis scores of indicated mice as in $\mathbf{d}\left({ }^{\star \star} P=0.0011\right)$. f ILC3s $\left(3 \times 10^{4}\right.$ cells per mouse) isolated from $\mathrm{Mbd3}^{-/-}$, circKcnt2 ${ }^{-/-}$, circKcnt2 ${ }^{-/}$- Batf ${ }^{-/-}$(DKO), or WT control mice were transplanted into $\mathrm{Rag}^{-/} / 1 / 2 \mathrm{rg}^{-/-}$mice. Two weeks later, mice were administrated with DSS and anti-IL-17 or isotype IgG. Colons were isolated and analyzed by H\&E staining. Scale bar, $100 \mu \mathrm{m}$. $\mathbf{g}$ Colitis scores of indicated mice as in $\mathbf{f}\left({ }^{\star} P=0.0219\right.$; $\left.{ }^{\star \star \star} P=0.0006\right) . n=5$ independent samples. ${ }^{\star} P<0.05$ and ${ }^{\star \star} P<0.01$. Data are shown as means \pm SD. Data were analyzed by an unpaired Student's $t$-test and representative of at least three independent experiments. Source data are provided as a Source Data file.

ILC3s to produce IL-22, which is essential for intestinal barrier integrity and mucosal healing ${ }^{37}$. IL-22 deficiency of ILC3s causes severe inflammation during Citrobacter rodentium infection or DSS-induced acute colitis ${ }^{6}$. TL1A derived from CX3CR $1^{+}$ mononuclear phagocytes synergizes with IL-23 to enhance IL-22 secretion in ILC $3 s^{6}$. On the other hand, regulatory T cells indirectly restrain ILC3 function to alleviate colitis via inhibiting production of IL-23 and IL- $1 \beta$ by CX3CR $1^{+}$macrophages $^{12}$. We previously demonstrated that ILCregs directly produce IL-10 to suppress ILC3 activation to facilitate resolution of intestinal inflammation ${ }^{13}$. As for intrinsic regulation, RANKL is reported to suppress ILC3 activity in the intestine via RANK-RANKL interactions between ILC $3 s^{5}$. However, how other intrinsic factors regulate ILC3 activity still remains unclear. Herein we identified an inducible circRNA circKcnt2 in ILC3s during intestinal inflammation, which suppresses ILC3 activation to promote resolution of intestinal colitis. Our findings reveal that circRNAs played a crucial role in the regulation of ILC3 activity as intrinsic factors.

CircRNAs are a new class of noncoding RNA that are formed via covalent linkage of single RNA molecules by backsplicing ${ }^{14,38}$. CircRNAs are resistant to RNA exonucleases and possesses a longer half-life than other RNAs. Accumulating evidence indicates that circRNAs participate in the regulation of various biological processes via several mechanisms ${ }^{39,40}$.
Some circRNAs specifically bind miRNAs as miRNA sponges to regulate gene expression ${ }^{19,20}$. In addition, some circRNAs exist in the nucleus and could regulate gene transcription ${ }^{21,41}$. We recently showed that circRNA cia-cGAS resides in the nuclei of HSCs to block the enzymatic activity of cGAS, which protects dormant HSCs from cGAS-mediated exhaustion ${ }^{18}$. We also identified circRNA circPan3 is highly expressed in intestinal stem cells (ISCs), which binds Il13ral mRNA to increase its stability leading to the expression of IL-13R 1 1 in ISCs for IL-13-IL-13Rmediated self-renewal maintenance of ISCs ${ }^{23}$. In this study, we showed that circKcnt2 is upregulated in the nucleus of ILC3s over intestinal inflammation. CircKcnt2 recruits the NuRD complex onto Batf promoter to suppress its expression, which inhibits ILC3 activation to facilitate resolution of innate colitis. Of note,

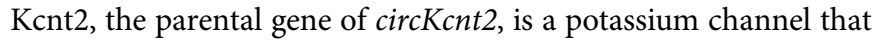
specifically expresses in pain-sensing dorsal root ganglia neurons to reduce neuronal excitability ${ }^{42}$. We noticed that circKcnt2 deletion does not affect Kcnt2 expression and Kcnt2 knockout has no effect on colitis induction, suggesting that circKcnt2 exerts an independent role versus its parental gene.

ILC functions are finely regulated by both genetic and epigenetic aspects ${ }^{43}$. Chromatin remodeling is a prerequisite for eukaryotic gene transcription, depending on ATP-dependent remodeling complexes (also known as remodelers) ${ }^{44}$. These remodelers 
modulate chromatin structures via multiple means, including histone modifications, DNA methylations, and incorporation of histone variants. We previously showed that the Pcid2 controls lymphoid lineage development via modulating the activity of SRCAP remodeling complex ${ }^{45}$. We also demonstrated that the NURF remodeling complex is involved in the regulation of ILC maintenance ${ }^{4}$. The NuRD (nucleosome remodeling and deacetylase) complex, as a transcriptional repressor, forms repressive chromatin structure via the nucleosome remodeling $(\mathrm{Mi}-2 \alpha / \beta$ subunits) and histone deacetylase (HDAC1/2 subunits) activities $^{46,47}$. Mbd3 deletion causes a defect of embryonic stem (ES) cell differentiation ${ }^{32,48}$. We previously found that Sox2 recruits the NuRD complex to inhibit mTOR expression, which drives autophagy induced cellular reprogramming 49 . Herein we showed that circKcnt2 directly interacts with Mbd3 in ILC3s that recruits the NuRD complex onto Batf promoter, leading to inhibition of Batf transcription. Furthermore, Mbd3 deficiency causes severe colitis after DSS treatment. Our findings reveal that circRNAs-mediated chromatin remodeling by the NuRD complex plays a critical role in the regulation of ILC3 function.

Batf, a member of BATF family, is a basic leucine zipper TF that regulates gene transcription via associating with Jun proteins ${ }^{25}$. The functional investigations of Batf were previously mainly focused on T cells. Batf is involved in the development and differentiation of several types of $\mathrm{T}$ cells, such as Th2 and Th17 cells ${ }^{26,33}$. In addition, Batf is also implicated in the regulation of $\mathrm{T}$ cell functions. For instance, Batf enhances development of colitis-associated colon cancer relying on Th17 cells ${ }^{50}$. Batf also regulates intestinal graft-versus-host disease via IL$7 \mathrm{R}^{\text {hi GM-CSF }}{ }^{+} \mathrm{T}$ cells ${ }^{51}$. Moreover, Batf deficiency causes reduced numbers of effector $\mathrm{T}$ and regulatory $\mathrm{T}$ cells in the intestine $\mathrm{e}^{52}$. Here, we found that Batf promotes IL-17 expression of ILC3s to initiate ILC3 activation. Batf deletion suppresses ILC3 activation and attenuates innate colitis. In sum, circKcnt2 recruits the NuRD complex onto Batf promoter in ILC3s to repress Batf transcription, which suppresses ILC3 activation and facilitate resolution of innate colitis.

\section{Methods}

Antibodies and reagents. Anti-H3K27ac (Cat\# 8173), anti-Batf (Cat\# 8638), antiH3K27me3 (Cat\# 9733), anti-Mbd3 (Cat\# 99169), anti-Chd3 (Cat\# 4241), antiRbap46 (Cat\# 6882), anti-Hdacl (Cat\# 34589), anti-Hdac2 (Cat\# 57156), antiEEA1 (Cat\# 3288), anti-H3 (Cat\# 4499), and anti- $\beta$-actin (Cat\# 3700) were from Cell Signaling Technology (Danvers, USA). Anti-Kcnt2 (Cat\# bs-12177R) was from Bioss Antibodies (Beijing, China). Anti-CD127 (A7R34), anti-c-Kit (2B8), antiCD3 (17A2), anti-CD4 (GK1.5), anti-CD19 (1D3), anti-NK1.1 (PK136), antiCD150 (mShad150), anti-CD34 (RAM34), anti-CD45 (30-F11), anti-CD90 (HIS51), anti-Sca-1 (D7), anti-CD25 (PC61.5), anti-Flt3 (A2F10), anti- $\alpha_{4} \beta_{7}$ (DATK32), anti-ROR $\gamma \mathrm{t}$ (AFKJS-9), anti-NKp46 (29A1.4), anti-Gata3 (TWAJ), anti-KLRG1 (2F1), anti-PLZF (Mags.21F7), Lineage cocktail (88-7772-72), antiCD48 (HM48-1), Anti-IL-17 (eBio17B7), and anti-CD16/32 (93) were purchased from eBiosciences (San Diego, USA). Anti-BrdU (600-401-C29) was purchased from ThermoFisher. Paraformaldehyde (PFA) and 4',6-diamidino-2-phenylindole (DAPI) were from Sigma-Aldrich. The IL-17 ELISA kit was purchased from eBiosciences.

Generation of knockout mice by CRISPR/Cas9 technology. For generation of circKcnt2 $2^{-1-}$, Batf $^{-1-}$, circKcnt $2^{\text {Mut }}$, and Kcnt2 ${ }^{-1-}$ mice, CRISPR-mediated singlestranded oligodecxynucleotides donors were synthesized as previous described ${ }^{53}$. About 250 zygotes from C57BL/6 mice were injected with sgRNAs and subsequently transferred to the uterus of pseudo-pregnant ICR females from which viable founder mice were obtained. Genomic DNA mutation was identified by PCR screening and DNA sequencing, followed by western blotting or northern blotting. sgRNA sequences are listed in Supplementary Table 2. Mbd3 flox/flox mice were a gift from Dr. Brian Hendrich (University of Cambridge, UK). Rorc-Cre (022791), PLZFGFPcre (024529), Rorc $(\gamma \mathrm{t})^{+/ G F P}(007572)$, and Id2 ${ }^{+/ G F P}(016224)$ mice were purchased from the Jackson Laboratory. Il10-l- (J002251) mice were purchased from Model Animal Research Center of Nanjing University, China. Mbd3 flox/floxRorc-Cre mice were obtained by crossing $M b d 3^{\text {flox/flox }}$ mice with Rorc-Cre mice.

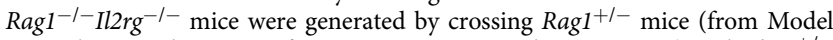
Animal Research Center of Nanjing University, China; J002216) with Il2rg $\mathrm{rg}^{+/-}$ mice. All the mouse strains were of C57BL/6 background and were maintained under specific pathogen-free conditions with approval by the Institutional Committee of Institute of Biophysics, Chinese Academy of Sciences. The study is compliant with all relevant ethical regulations regarding animal research.

Intestinal lymphocyte separation. Intestinal lymphocytes were obtained as previously reported ${ }^{4}$. Briefly, the Peyer's patches were removed from the small intestine. Then the intestine was cut open longitudinally, followed by wash using phosphate buffer saline (PBS) five times. Afterwards, the intestine was cut into pieces and washed using solution I buffer (10 mM HEPES and $5 \mathrm{mM}$ EDTA in HBSS) five times, followed by digestion using solution II buffer (DNaseI, 5\% FBS, $0.5 \mathrm{mg} / \mathrm{ml}$ collagenase II and collagenase III) three times. Finally, LPLs were sifted through $70 \mu \mathrm{m}$ strainers and utilized for experiments.

Flow cytometry. BM cells were flushed out from femurs in PBS (containing 5\% FBS) buffer and sifted through $70 \mu \mathrm{m}$ cell strainers. LPL cells were isolated as described above. For flow cytometric analysis, LT-HSC $\left(\mathrm{Lin}^{-} \mathrm{Sca}-1^{+} \mathrm{c}-\mathrm{Kit}^{+} \mathrm{CD} 150^{+}\right.$ CD48 $\left.{ }^{-}\right)$, ST-HSC $\left(\mathrm{Lin}^{-} \mathrm{Sca}-1^{+} \mathrm{c}-\mathrm{Kit}^{+} \mathrm{CD} 150^{-} \mathrm{CD} 48^{-}\right)$, CMP $\left(\mathrm{Lin}^{-} \mathrm{Sca}-1^{-} \mathrm{c}-\mathrm{Kit}^{+}\right.$

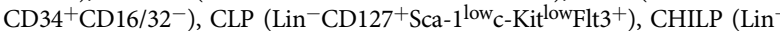

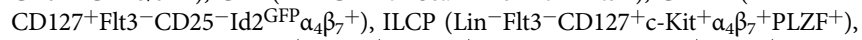
ILC1 $\left(\mathrm{CD} 3^{-} \mathrm{CD} 19^{-} \mathrm{CD} 127^{+} \mathrm{NK} 1.1^{+} \mathrm{NKp} 46^{+}\right)$, ILC2 $\left(\mathrm{Lin}^{-} \mathrm{CD} 127^{+}{ }^{-} \mathrm{CD} 90^{+} \mathrm{KLRG}^{+}\right.$ Gata3 $^{+}$), ILC3 $\left(\mathrm{Lin}^{-} \mathrm{ROR} \gamma \mathrm{t}^{+} \mathrm{CD} 45^{\text {low }} \mathrm{CD} 127^{+}\right.$or $\left.\mathrm{Lin}^{-} \mathrm{CD} 90^{\text {high }} \mathrm{CD} 45^{\text {low }}\right)$, NK $\left(\mathrm{CD}^{-}{ }^{-} \mathrm{CD} 19^{-} \mathrm{CD}^{2} 7^{-} \mathrm{NK} 1.1^{+}\right), \mathrm{CD}^{+} 9^{+} \mathrm{B}$, and $\mathrm{CD}^{+} \mathrm{T}$ populations were sorted with a FACSAria III instrument (BD Biosciences). PLZFGFPcre mice were used for ILCPs isolation and $I d 2^{+/ G F P}$ mice were used for CHILPs isolation by FACS.

Immunofluorescence staining. ILC3s ( $\mathrm{Lin}^{-} \mathrm{CD} 90^{\text {high }} \mathrm{CD} 45^{\text {low }}$ ) isolated by FACS were fixed with $4 \%$ PFA (Sigma-Aldrich) for $20 \mathrm{~min}$ at room temperature, perforated with PBS containing 1\% TritonX-100 for 20 min, blocked with 5\% donkey and $5 \%$ rat serum for $1 \mathrm{~h}$ at room temperature, incubated with appropriate primary antibodies at $4{ }^{\circ} \mathrm{C}$ overnight, and then incubated with fluorescence-conjugated secondary antibodies. DAPI was used for nucleus staining. Cells were visualized with Olympus FV1200 laser scanning confocal microscopy (Olympus, Japan).

DNA fluorescence in situ hybridization. ILC3s ( $\left.\mathrm{Lin}^{-} \mathrm{CD} 90^{\text {high }} \mathrm{CD} 45^{\text {low }}\right)$ were isolated and fixed with $4 \%$ PFA containing $10 \%$ acetic acid for $20 \mathrm{~min}$ at room temperature. Then cells were treated with $70 \%$ ethanol at $-20{ }^{\circ} \mathrm{C}$ and incubated in buffer containing $100 \mathrm{mM}$ Tris- $\mathrm{HCl}(\mathrm{pH} 7.5), 150 \mathrm{mM} \mathrm{NaCl}$, followed by cytoplasm digestion in $0.01 \%$ pepsin $/ 0.01 \mathrm{~N} \mathrm{HCl}$ for $3 \mathrm{~min}$ at $37^{\circ} \mathrm{C}$. Cells were further fixed with $3.7 \%$ PFA and replaced with ethanol to a final concentration of $100 \%$. Afterwards, cells were air dried and washed using $2 \times$ SSC and blocked using buffer containing $100 \mathrm{mM}$ Tris- $\mathrm{HCl}$ (pH 7.5), $150 \mathrm{mM} \mathrm{NaCl}, 0.05 \%$ Tween 20, 3\% BSA for $20 \mathrm{~min}$. Then, cells were denatured in $70 \%$ formamide $/ 2 \times$ SSC and incubated with fluorescence-labeled DNA probes overnight. After staining with DAPI for nucleus, cells were visualized by Olympus FV1200 laser scanning confocal microscopy (Olympus, Japan).

Real-time quantitative PCR. RNAs were extracted using a RNA Miniprep Kit (Tiangen, Beijing, China) according to the manufacturer's protocol as previously described $^{54}$. Then cDNA was synthesized with M-MLV reverse transcriptase (Promega, Madison, USA). Gene expression was analyzed on an ABI 7300 qPCR system using specific primer pairs listed in Supplementary Table 3. Relative expression levels were calculated and normalized to endogenous $18 \mathrm{~S}$ or Gapdh.

Chromatin immunoprecipitation assay. ILC3s were cross-linked with $1 \%$ formaldehyde at $37^{\circ} \mathrm{C}$ for $10 \mathrm{~min}$. Then cells were washed twice with PBS, lysed with SDS lysis buffer (1\% SDS, $10 \mathrm{mM}$ EDTA, $50 \mathrm{mM}$ Tris), and sonicated to make 200-500 bp DNA fragments. Lysates were pre-cleared with Protein A Agarose/ Salmon Sperm DNA (50\% Slurry) and then incubated with $4 \mu \mathrm{g}$ antibody overnight at $4{ }^{\circ} \mathrm{C}$. Then Protein A Agarose/Salmon Sperm DNA (50\% Slurry) beads were added and incubated for $4 \mathrm{~h}$. After washing, DNA was eluted from beads and purified. DNA fragments were analyzed using primer pairs listed in Supplementary Table 4. For ChIP immunoblotting assay, LPL cells were cross-linked with $1 \%$ formaldehyde and lysed with SDS lysis buffer and sonicated to 200-500 bp. Lysates were pre-cleared with protein A agarose/salmon sperm DNA ( $50 \%$ slurry). Then, 4 $\mu \mathrm{g}$ antibody was incubated with treated lysates for ChIP assays, followed by size fractionation with sucrose gradient ultracentrifugation. Five hundred microliters eluate was put onto $30 \mathrm{ml} \mathrm{5-30 \%} \mathrm{(V/V)} \mathrm{sucrose} \mathrm{gradient} \mathrm{followed} \mathrm{by} \mathrm{ultra-}$ centrifugation at 55,000 $g$ with an S Beckman SW28 rotor. Eluent gradients were concentrated and reversely cross-linked. Proteins were examined by western blotting. DNA was extracted and examined by PCR assays.

DSS-induced colitis. For DSS-induced colitis, indicated mice (about 8 weeks) were administered 3\% DSS in drinking water for 7 days, followed by 7 days of normal water. 
Helicobacter hepaticus infection. Helicobacter hepaticus, Hh NCI-Frederick isolate 1A (strain 51449; American Type Culture Collection), was used for colitis induction as previously reported ${ }^{9}$. In brief, H.h. was grown on blood agar plates containing trimethoprim, vancomycin, and polymyxin B (all from Oxoid) under microaerophilic conditions. For H.h. infection, indicated mice were infected with $1 \times 10^{8}$ colony-forming unit H.h. by oral gavage for three times on alternate days. Colitis was analyzed 8 weeks later after the first $H$. hepaticus inoculation. For quantitation of H.h., DNA was purified from cecal contents taken from H. hepaticus-infected mice using the DNA Stool kit (Qiagen). H.h. DNA was determined using a Q-PCR method as previously described ${ }^{55}$.

Colitis scores and histologic analysis. Colons were cut open longitudinally and fixed in $4 \%$ PFA followed by paraffin sectioning and H\&E staining. The colitis score was calculated according to weight loss, appearance of the stool, intestinal bleeding, and histology as reported before ${ }^{56}$.

Adoptive transfer assay. $3 \times 10^{4}$ ILC3s were adoptively transferred into Rag1 $1^{-/-}$ Il2rg ${ }^{-1-}$ mice. Two weeks later, transferred mice were treated with 3\% DSS and colitis was then analyzed as described above.

Lentivirus preparation and infection. RNA interference was performed as previously described ${ }^{18}$. And target sequences are listed in the Supplementary Table 5. For circKcnt2 overexpression, genomic exon regions of $\operatorname{circKcnt2}$ were constructed into split GFP site on pSIN-EF2-GFP vector flanked with the upstream and downstream complementary elements. Lentiviral vector (pSIN-EF2-GFP) was cotransfected with packaging plasmids pVSVg and psPAX2 into HEK293T (ATCC ${ }^{\circledR}$ CRL-11268 ${ }^{\text {tw }}$ ) cells for $48 \mathrm{~h}$, followed by culture medium collection and ultracentrifugation at $25,000 \mathrm{~g}$ for $1.5 \mathrm{~h}$. Pellets were resuspended in IMDM medium and viral titers were determined by infecting HEK293T cells with diluted viruses. Cells were incubated with lentiviruses $(\mathrm{MOI}=10)$ and centrifuged at $500 \mathrm{~g}$ for $2 \mathrm{~h}$ in the presence of $8 \mu \mathrm{g} / \mathrm{ml}$ polybrene. Cells were cultured for $24 \mathrm{~h}$ to allow GFP expression, followed by sorting of GFP-positive cells through a flow cytometer. HEK293T cells were from ATCC (CRL-11268) and tested negative for mycoplasma contamination.

Immunoprecipitation assay. LPL cells were lysed and supernatants were incubated with biotin-labeled circKcnt2 or antisence. Then precipitated components were separated with SDS-PAGE and silver staining. Differential bands enriched by circKcnt2 were analyzed by LTQ Orbitrap XL mass spectrometry or immunoblotting.

Nuclear run-on assay. Sorted cells were harvested in buffer containing $150 \mathrm{mM}$ $\mathrm{KCl}, 10 \mathrm{mM}$ Tris- $\mathrm{HCl}, 4 \mathrm{mM} \mathrm{MgOAc}$ with $\mathrm{pH}$ 7.4, followed by centrifugation to collect cell pellets. Pellets were lysed in buffer containing $150 \mathrm{mM} \mathrm{KCl}, 10 \mathrm{mM}$ Tris- $\mathrm{HCl}, 4 \mathrm{mM} \mathrm{MgOAc}$, and $0.5 \% \mathrm{NP}-40$, followed by sucrose density gradient centrifugation to prepare crude nuclei. Crude nuclei were incubated with $10 \mathrm{mM}$ ATP, CTP, GTP, BrUTP, and RNase inhibitor at $28^{\circ} \mathrm{C}$ for $5 \mathrm{~min}$. RNAs were extracted using TRIzol reagent with manufacturer's guidelines, followed by DNA digestion with DNaseI. RNA transcripts were immunoprecipitated with antibody against BrdU, followed by reverse transcription and RT-PCR analysis. $5 \times 10^{4}$ cells were used for each single sample.

Microarray assay. For circRNA microarray, ILC3s ( $\left.\mathrm{Lin}^{-} \mathrm{CD} 90^{\text {high }} \mathrm{CD} 45^{\text {low }}\right)$ were isolated from $R a g 1^{-1-}$ mice treated with or without DSS. Then RNAs were isolated using Trizol reagent (Invitrogen) and used for Mouse circular RNA Array V2.0 assay (GSE142106). For mRNA microarray, RNAs from $c i r c \mathrm{Kcnt}_{2}{ }^{+l}$ and circKcnt2 $^{-1-}$ ILC3s were isolated using Trizol reagent (Invitrogen) and prepared for Affymetrix mRNA microarray assay (GSE142766) by Beijing Cnkingbio Biotechnology.

RNA hybridization. Northern blotting was performed as previously described 4 . Briefly, after extracted using TRIzol, total RNA was subjected to formaldehydedenaturing agarose electrophoresis, followed by transfer to positively charged nitrocellulose (NC) membrane with $20 \times \mathrm{SSC}$ buffer $(3.0 \mathrm{M} \mathrm{NaCl}$ and $0.3 \mathrm{M}$ sodium citrate, $\mathrm{pH}$ 7.0). Then, the membrane was UV cross-linked and incubated with hybrid buffer for $2 \mathrm{~h}$ prehybridization, and then with biotin-labeled RNA probes (targeting circKcnt2: nt 262-127). Biotin signals were detected with HRPconjugated streptavidin according to the manufacturer's instructions (Thermo Scientific). For dot blotting, RNA was dropped onto Hybond $-\mathrm{N}^{+}$membrane (GE Healthcare), followed by UV cross-linking. Then RNA signal was detected using biotin-labeled single-stranded DNA segment. RNA was generated by in vitro transcription.

Fluorescence in situ hybridization. ILC3s were hybridized with fluorescenceconjugated $\operatorname{circKcnt} 2$ probes (5'-ACCCAAAATATGTGAGACCAAGTGAAGATA AGGCA-3') and then immune-stained with appropriate antibodies. Images were obtained with an Olympus FV1000 laser scanning confocal microscope.
DNasel accessibility assay. Nuclei were purified from ILC3s according to the manufacturer's protocol with a nuclei-isolation kit (Sigma-Aldrich). Then nuclei were resuspended with DNaseI digestion buffer and treated with DNaseI (Sigma) at $37^{\circ} \mathrm{C}$ for $5 \mathrm{~min}$. Then $2 \times$ DNaseI stop buffer $(20 \mathrm{mM}$ Tris, $\mathrm{pH} 8.0,4 \mathrm{mM}$ EDTA, 2 mM EGTA) was added to stop reactions. DNA was extracted and measured by qPCR.

Electrophoretic mobility shift assay. Biotin-labeled linearized circKcnt2 were synthesized by T7 transcription in vitro. Probes and Mbd3 proteins were incubated in binding buffer and mobility shift assay was performed using a Light Shift Chemiluminescent RNA EMSA Kit (Thermo Scientific) according to the manufacturer's protocol.

ELISA assay. ILC3s were isolated and cultured for $24 \mathrm{~h}$ with indicated cytokines Then supernatants were collected and cytokines were detected using ELISA kit (eBioscience) according to the manufacturer's instructions.

Statistical analysis. Data were analyzed by GraphPad Prism 6.0. All flow cytometry data were analyzed with FlowJo 7. Adobe Photoshop CC 14.0 and ImageJ 1.48 were used for figure presentation. For statistical evaluation, an unpaired Student's $t$-test was applied for calculating statistical probabilities in this study. For al panels, at least three independent experiments were performed with similar results, and representative experiments are shown. Data were presented as mean \pm SD. $P$ values $\leq 0.05$ were considered significant $\left({ }^{*} P<0.05 ;{ }^{*} P<0.01 ;{ }^{* * *} P<0.001\right)$; $P>0.05$, non-significant (NS)

Reporting summary. Further information on research design is available in the Nature Research Reporting Summary linked to this article.

\section{Data availability}

The circRNA and mRNA microarray data were deposited to the GEO database (GSE142106 and GSE142766). All other data are included in the supplementary information or available from the authors upon reasonable requests. Source data are provided with this paper.

Received: 18 September 2019; Accepted: 28 July 2020; Published online: 14 August 2020

\section{References}

1. Eberl, G., Colonna, M., Di Santo, J. P. \& McKenzie, A. N. Innate lymphoid cells: a new paradigm in immunology. Science 348, aaa6566 (2015).

2. Diefenbach, A., Colonna, M. \& Koyasu, S. Development, differentiation, and diversity of innate lymphoid cells. Immunity 41, 354-365 (2014).

3. Serafini, N., Vosshenrich, C. A. \& Di Santo, J. P. Transcriptional regulation of innate lymphoid cell fate. Nat. Rev. Immunol. 15, 415-428 (2015).

4. Liu, B. et al. Long noncoding RNA $\operatorname{lncKdm} 2 b$ is required for ILC3 maintenance by initiation of Zfp292 expression. Nat. Immunol. 18, 499-508 (2017).

5. Bando, J. K. et al. The tumor necrosis factor superfamily member RANKI suppresses effector cytokine production in group 3 innate lymphoid cells. Immunity 48, 1208-1219. e1204 (2018).

6. Castellanos, J. G. et al. Microbiota-Induced TNF-like ligand 1A drives group 3 innate lymphoid cell-mediated barrier protection and intestinal $\mathrm{T}$ cell activation during colitis. Immunity 49, 1077-1089.e1075 (2018).

7. Dalli, J., Colas, R. A., Arnardottir, H. \& Serhan, C. N. Vagal regulation of group 3 innate lymphoid cells and the immunoresolvent PCTR1 controls infection resolution. Immunity 46, 92-105 (2017).

8. Kathania, M. et al. Itch inhibits IL-17-mediated colon inflammation and tumorigenesis by ROR-gammat ubiquitination. Nat. Immunol. 17, 997-1004 (2016).

9. Buonocore, S. et al. Innate lymphoid cells drive interleukin-23-dependent innate intestinal pathology. Nature 464, 1371-1375 (2010).

10. Wirtz, S. et al. Chemically induced mouse models of acute and chronic intestinal inflammation. Nat. Protoc. 12, 1295-1309 (2017).

11. Guo, X. et al. Induction of innate lymphoid cell-derived interleukin-22 by the transcription factor STAT3 mediates protection against intestinal infection. Immunity 40, 25-39 (2014).

12. Bauche, D. et al. LAG3 $(+)$ regulatory T cells restrain interleukin-23producing $\mathrm{CX} 3 \mathrm{CR} 1(+)$ gut-resident macrophages during group 3 innate lymphoid cell-driven colitis. Immunity 49, 342-352.e345 (2018).

13. Wang, S. et al. Regulatory innate lymphoid cells control innate intestinal inflammation. Cell 171, 201-216.e218 (2017).

14. Chen, L. L. The biogenesis and emerging roles of circular RNAs. Nat. Rev. Mol. Cell Biol. 17, 205-211 (2016). 
15. Vicens, Q. \& Westhof, E. Biogenesis of circular RNAs. Cell 159, 13-14 (2014).

16. Zhang, X. O. et al. Complementary sequence-mediated exon circularization. Cell 159, 134-147 (2014).

17. Conn, S. J. et al. The RNA binding protein quaking regulates formation of circRNAs. Cell 160, 1125-1134 (2015).

18. Xia, P. et al. A circular RNA protects dormant hematopoietic stem cells from DNA sensor cGAS-mediated exhaustion. Immunity 48, 688-701.e687 (2018).

19. Memczak, S. et al. Circular RNAs are a large class of animal RNAs with regulatory potency. Nature 495, 333-338 (2013).

20. Hansen, T. B. et al. Natural RNA circles function as efficient microRNA sponges. Nature 495, 384-388 (2013).

21. Li, Z. et al. Exon-intron circular RNAs regulate transcription in the nucleus. Nat. Struct. Mol. Biol. 22, 256-264 (2015).

22. Vo, J. N. et al. The landscape of circular RNA in cancer. Cell 176, 869881.e813 (2019).

23. Zhu, P. et al. IL-13 secreted by ILC2s promotes the self-renewal of intestinal stem cells through circular RNA circPan3. Nat. Immunol. 20, 183-194 (2019).

24. Tussiwand, R. et al. Compensatory dendritic cell development mediated by BATF-IRF interactions. Nature 490, 502-507 (2012).

25. Murphy, T. L., Tussiwand, R. \& Murphy, K. M. Specificity through cooperation: BATF-IRF interactions control immune-regulatory networks. Nat. Rev. Immunol. 13, 499-509 (2013).

26. Betz, B. C. et al. Batf coordinates multiple aspects of B and $\mathrm{T}$ cell function required for normal antibody responses. J. Exp. Med. 207, 933-942 (2010).

27. Kurachi, M. et al. The transcription factor BATF operates as an essential differentiation checkpoint in early effector CD8+ T cells. Nat. Immunol. 15, 373-383 (2014).

28. Quigley, M. et al. Transcriptional analysis of HIV-specific CD8+ T cells shows that PD-1 inhibits T cell function by upregulating BATF. Nat. Med. 16, 1147-1151 (2010).

29. Vasanthakumar, A. et al. The transcriptional regulators IRF4, BATF and IL-33 orchestrate development and maintenance of adipose tissue-resident regulatory T cells. Nat. Immunol. 16, 276-285 (2015).

30. Hayatsu, N. et al. Analyses of a mutant Foxp3 allele reveal BATF as a critical transcription factor in the differentiation and accumulation of tissue regulatory T cells. Immunity 47, 268-283.e269 (2017).

31. Mazmanian, S. K., Round, J. L. \& Kasper, D. L. A microbial symbiosis factor prevents intestinal inflammatory disease. Nature 453, 620-625 (2008).

32. dos Santos, R. L. et al. MBD3/NuRD facilitates induction of pluripotency in a context-dependent manner. Cell Stem Cell 15, 102-110 (2014).

33. Schraml, B. U. et al. The AP-1 transcription factor Batf controls $\mathrm{T}(\mathrm{H}) 17$ differentiation. Nature 460, 405-409 (2009).

34. Artis, D. \& Spits, H. The biology of innate lymphoid cells. Nature 517, 293-301 (2015).

35. Guo, X. et al. Innate lymphoid cells control early colonization resistance against intestinal pathogens through ID2-dependent regulation of the microbiota. Immunity 42, 731-743 (2015).

36. Kirchberger, S. et al. Innate lymphoid cells sustain colon cancer through production of interleukin-22 in a mouse model. J. Exp. Med. 210, 917-931 (2013).

37. Longman, R. S. et al. CX(3)CR1(+) mononuclear phagocytes support colitisassociated innate lymphoid cell production of IL-22. J. Exp. Med. 211, 1571-1583 (2014).

38. Petkovic, S. \& Muller, S. RNA circularization strategies in vivo and in vitro. Nucleic Acids Res. 43, 2454-2465 (2015).

39. Liu, C. X. et al. Structure and degradation of circular RNAs regulate PKR activation in innate immunity. Cell 177, 865-880.e821 (2019).

40. Wesselhoeft, R. A. et al. RNA circularization diminishes immunogenicity and can extend translation duration in vivo. Mol. Cell 74, 508-520. e504 (2019).

41. Zhang, Y. et al. Circular intronic long noncoding RNAs. Mol. Cell 51, 792-806 (2013).

42. Tsantoulas, C. \& McMahon, S. B. Opening paths to novel analgesics: the role of potassium channels in chronic pain. Trends Neurosci. 37, 146-158 (2014).

43. Antignano, F. et al. G9a regulates group 2 innate lymphoid cell development by repressing the group 3 innate lymphoid cell program. J. Exp. Med. 213, 1153-1162 (2016).

44. Krasteva, V. et al. The BAF53a subunit of SWI/SNF-like BAF complexes is essential for hemopoietic stem cell function. Blood 120, 4720-4732 (2012).

45. Ye, B. et al. Suppression of SRCAP chromatin remodelling complex and restriction of lymphoid lineage commitment by Pcid2. Nat. Commun. 8, 1518 (2017).

46. Aguilera, C. et al. c-Jun N-terminal phosphorylation antagonises recruitment of the Mbd3/NuRD repressor complex. Nature 469, 231-235 (2011).

47. Hu, G. et al. H2A.Z facilitates access of active and repressive complexes to chromatin in embryonic stem cell self-renewal and differentiation. Cell Stem Cell 12, 180-192 (2013).
48. Yildirim, O. et al. Mbd3/NURD complex regulates expression of 5hydroxymethylcytosine marked genes in embryonic stem cells. Cell 147, 1498-1510 (2011).

49. Wang, S. et al. Transient activation of autophagy via Sox2-mediated suppression of mTOR is an important early step in reprogramming to pluripotency. Cell Stem Cell 13, 617-625 (2013).

50. Punkenburg, E. et al. Batf-dependent Th17 cells critically regulate IL-23 driven colitis-associated colon cancer. Gut 65, 1139-1150 (2016).

51. Ullrich, E. et al. BATF-dependent IL-7RhiGM-CSF+ T cells control intestinal graft-versus-host disease. J. Clin. Invest. 128, 916-930 (2018).

52. Wang, C. et al. BATF is required for normal expression of gut-homing receptors by $\mathrm{T}$ helper cells in response to retinoic acid. J. Exp. Med. 210, 475-489 (2013).

53. Zhu, X. et al. An efficient genotyping method for genome-modified animals and human cells generated with CRISPR/Cas9 system. Sci. Rep. 4, 6420 (2014)

54. Liu, B. et al. IL-7Ralpha glutamylation and activation of transcription factor Sall3 promote group 3 ILC development. Nat. Commun. 8, 231 (2017).

55. Ge, Z., White, D. A., Whary, M. T. \& Fox, J. G. Fluorogenic PCR-based quantitative detection of a murine pathogen, Helicobacter hepaticus. J. Clin. Microbiol. 39, 2598-2602 (2001).

56. Gagliani, N. et al. Th17 cells transdifferentiate into regulatory $\mathrm{T}$ cells during resolution of inflammation. Nature 523, 221-225 (2015).

\section{Acknowledgements}

We thank Shu Meng, Dongdong Fan, Yan Teng, Junying Jia, and Xiang Shi for technical support. We also thank Jing Li (Cnkingbio Company Ltd, Beijing, China) for technical support. This work was supported by National Natural Science Foundation of China (31930036, 81921003, 91940305, 91640203, 31530093, 31771638, 81772646, 81572433, $31870883,31871494,31670886,31771638)$, the Ministry of Science and Technology of China (2019YFA0508501), Strategic Priority Research Programs of the Chinese Academy of Sciences (XDA19050301, XDB19030203), Beijing Natural Science Foundation (7181006, 5192018), and the Young Elite Scientist Sponsorship Program by CAST (2018QNRC001).

\section{Author contributions}

B.L., B.Y., and Y.L. performed experiments; B.L. analyzed data and wrote the paper; X.Z constructed genetic mouse strains. H.L., N.L., P.Z., J.W., T.L., and L.H. analyzed data; T.Y. initiated the study and analyzed data; Z.F. initiated the study, organized, designed, and wrote the paper

\section{Competing interests}

The authors declare no competing interests.

\section{Additional information}

Supplementary information is available for this paper at https://doi.org/10.1038/s41467 020-17944-5.

Correspondence and requests for materials should be addressed to Y.T. or Z.F.

Peer review information Nature Communications thanks Eduardo Villablanca and the other, anonymous, reviewer(s) for their contribution to the peer review of this work.

Reprints and permission information is available at http://www.nature.com/reprints

Publisher's note Springer Nature remains neutral with regard to jurisdictional claims in published maps and institutional affiliations.

cc) (i) Open Access This article is licensed under a Creative Commons BY Attribution 4.0 International License, which permits use, sharing adaptation, distribution and reproduction in any medium or format, as long as you give appropriate credit to the original author(s) and the source, provide a link to the Creative Commons license, and indicate if changes were made. The images or other third party material in this article are included in the article's Creative Commons license, unless indicated otherwise in a credit line to the material. If material is not included in the article's Creative Commons license and your intended use is not permitted by statutory regulation or exceeds the permitted use, you will need to obtain permission directly from the copyright holder. To view a copy of this license, visit http://creativecommons.org/ licenses/by/4.0/.

(c) The Author(s) 2020 